\title{
Advances in Understanding Hydrogel Lubrication
}

\author{
Tooba Shoaib ${ }^{1}$ and Rosa M. Espinosa-Marzal ${ }^{1,2, *(\mathbb{D})}$ \\ 1 Department of Materials Science and Engineering, University of Illinois at Urbana-Champaign, \\ 1304 W Green St, Urbana, IL 61801, USA; shoaib2@illinois.edu \\ 2 Department of Civil and Environmental Engineering, University of Illinois at Urbana-Champaign, \\ Urbana, IL 61801, USA \\ * Correspondence: rosae@illinois.edu
}

Received: 19 September 2020; Accepted: 29 October 2020; Published: 13 November 2020

\begin{abstract}
Since their inception, hydrogels have gained popularity among multiple fields, most significantly in biomedical research and industry. Due to their resemblance to biological tribosystems, a significant amount of research has been conducted on hydrogels to elucidate biolubrication mechanisms and their possible applications as replacement materials. This review is focused on lubrication mechanisms and covers friction models that have attempted to quantify the complex frictional characteristics of hydrogels. From models developed on the basis of polymer physics to the concept of hydration lubrication, assumptions and conditions for their applicability are discussed. Based on previous models and our own experimental findings, we propose the viscous-adhesive model for hydrogel friction. This model accounts for the effects of confinement of the polymer network provided by a solid surface and poroelastic relaxation as well as the (non) Newtonian shear of a complex fluid on the frictional force and quantifies the frictional response of hydrogels-solid interfaces. Finally, the review delineates potential areas of future research based on the current knowledge.
\end{abstract}

Keywords: hydrogels; tribology; biolubrication; friction

\section{Introduction}

Tribosystems in nature, such as the oral cavity, the corneal epithelium and the articular cartilage provide exceptional life-long lubrication [1]. Biological tribosystems exhibit a biphasic composition, comprised of a macromolecular network consisting of mucins [2,3], polysaccharides [4], phospholipids [5], or glycoproteins [6], and a water-based lubricant [1]. Due to their compositional resemblance to biotribosystems, along with their hydrophilicity, biocompatibility and tunability of microstructure and chemical composition [7-9], hydrogels have gained popularity since their inception in 1960, both to study mechanisms underlying biolubrication, and as biomaterials, e.g., for cartilage replacement [10].

Figure 1 shows a schematic representation of a hydrogel's structure. Hydrogels are composed of chemically or physically crosslinked polymeric networks imbibed by a good solvent [11], in most cases an aqueous fluid. The blob-like structure of hydrogels is often described via the scaling laws proposed by de Gennes for polymer solutions in the semi-dilute regime. The distance between two crosslinks (labelled as mesh size, $\xi$ ) depends on the polymer concentration and the solvent quality [12,13], among others. This characteristic length determines key properties of hydrogels like permeability, elastic modulus and viscoelastic relaxation (Table 1). 


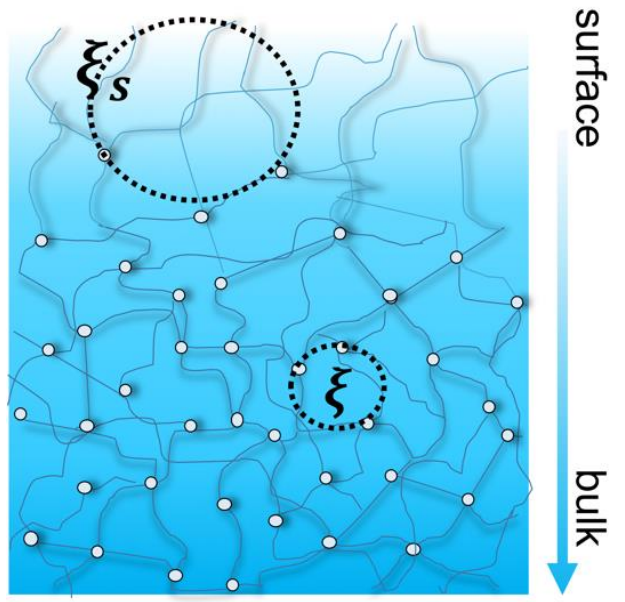

Figure 1. Hydrogel consisting of polymer chains permanently crosslinked or physically entangled. The mesh size $(\xi)$ (see dashed circles) may vary as a function of the hydrogel's depth due to gradients in crosslinking as well as polymer concentration.

Table 1. Characteristic properties of hydrogels: mesh size $(\xi)$, polymer relaxation time $\left(\tau_{A}\right)$, osmotic pressure (П) and poroelastic relaxation time $\left(\tau_{w}\right)$.

\begin{tabular}{|c|c|c|}
\hline Property & Equation & Ref. \\
\hline Mesh Size $(\xi)$ & $\begin{array}{l}\xi=\phi^{-\frac{v}{3 v-1}} \\
\text { where } \phi \text { is the polymer volume } \\
\text { fraction, and in semi-dilute regime; } \\
\qquad \begin{array}{l}v=3 / 5 \text { for good solvent; } \\
v=1 / 2 \text { for } \theta \text {-solvent. }\end{array}\end{array}$ & [13] \\
\hline Polymer relaxation time $\left(\tau_{A}\right)$ & $\begin{array}{c}\tau_{A} \sim \eta_{s} \xi^{3} / k_{B} T \\
\text { where } \eta_{s} \text { is the solvent viscosity, } \\
k_{B} \text { is the Boltzmann constant, } \\
\text { and } T \text { the temperature. }\end{array}$ & [13] \\
\hline Osmotic pressure (П) & $\begin{array}{c}\Pi \sim G^{\prime} \sim k_{B} T / \xi^{3} \\
\text { where } G^{\prime} \text { is the shear } \\
\text { storage modulus. }\end{array}$ & {$[13,14]$} \\
\hline Poroelastic relaxation time $\left(\tau_{w}\right)$ & $\begin{array}{l}\qquad \tau_{w} \sim \frac{a^{2}}{D} \sim \frac{6 \eta_{s} \pi a^{2}}{P \xi^{2}} \\
\text { where } a \text { is the contact radius, } \mathrm{D} \text { the } \\
\text { diffusivity and } P \text { the pressure. }\end{array}$ & [15] \\
\hline
\end{tabular}

Numerous studies have experimentally explored the load and speed dependence of the frictional response of hydrogels. Studies pioneered by Gong et al. showed that hydrogel friction does not follow Amonton's law, i.e., $F=\mu L$, where $F$ is the friction force, $L$ is the normal load, and $\mu$ the friction coefficient [16]. Subsequent studies showed that friction can either increase with load [17], be load-independent [16], or even decrease with increase in load [18,19]. A prominent dependence of friction on the sliding speed was reported concurrently and associated with viscous dissipation. This was initially attributed to the strong hydration of the gel, and thereby, to the possibility of sustaining hydrodynamic lubrication even at low sliding velocity and relatively high contact pressure. A dependence of friction on sliding velocity has been reported in many subsequent studies and over a wide range of conditions. Although these studies have also shown that hydrogel friction also varies with the chemical composition of the polymer [20-22], monomer and crosslinking concentrations [23,24], and surface roughness $[25,26]$, a detailed description of these results is out of the scope of this review.

A plethora of mechanisms have been proposed to explain the low friction coefficient provided by hydrogels. Commonalities among tribological studies include the relevance of distinct lubrication mechanisms at low and high sliding velocities. At high sliding velocities, friction results from the 
viscous dissipation upon shear of a fluid. Both hydrodynamic and non-hydrodynamic dissipative mechanisms arising from viscoelastic deformation have been proposed. At low sliding velocities, the frictional characteristics strongly depend on the adhesive vs. repulsive nature of the contact. It was Gong who first made the distinction between adhesive and non-adhesive (repulsive) contacts with hydrogels [27]. Additionally, the contact type (e.g., migrating vs. stationary contact) also plays a role in dictating frictional mechanism. In migrating contacts (e.g., a hard pin sliding on a hydrogel flat surface), a re-hydration of the interface and polymer relaxation are possible during the stress-free periods, while stationary contacts (e.g., a hydrogel microsphere sliding along a solid surface or a ring-on-disc tribometer) do not enable re-hydration of the near-surface region of the hydrogel (Figure 2). At small enough stroke lengths, reciprocating and unidirectional sliding have led to similar frictional characteristics of investigated hydrogels [28]. However, if the hydrogel surfaces have a texture or the surface structures are oriented/aligned in a particular direction, the sliding direction could affect the frictional response.

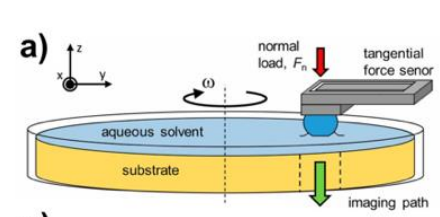

c)

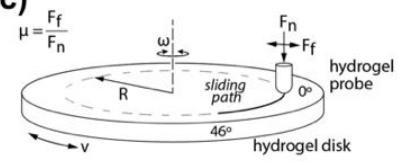

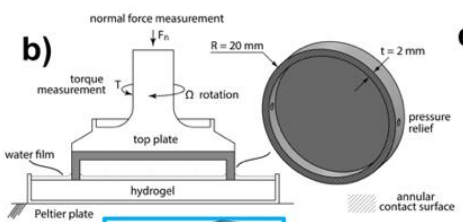

d)

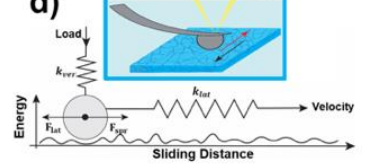

e)

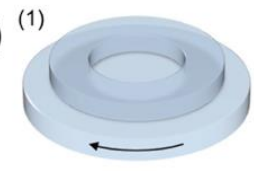

(3)

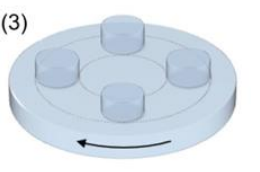

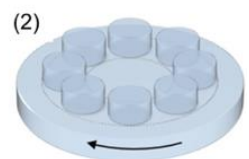

(4)

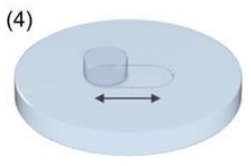

Figure 2. Schematics of typical contact configurations in friction force measurements on hydrogels. (a) Pin-on-disc tribometer, with the hydrogel as the spherical pin and a hard surface, which yields a static hydrogel-hard contact [29]. Reproduced with permission from Cuccia, N.L. et al., Pore-size dependence and slow relaxation of hydrogel friction on smooth surfaces. PNAS, 2020, 117, 11247-11256. (b) Rheometer providing an annular static contact [30]. Reprinted from Kim, J. and A.C. Dunn, Soft hydrated sliding interfaces as complex fluids. Soft Matter, 2016, 12, 6536-6546. (c) Pin-on-disc tribometer with the hydrogel as the flat substrate and a hard spherical pin as countersurface, yielding a migrating hydrogel-solid contact [31]. Reproduced with permission from Pitenis, A.A. et al., Polymer fluctuation lubrication in hydrogel Gemini interfaces. Soft Matter, 2014, 10, 8955-8962. (d) AFM setup for friction force measurement, with a silica microsphere (solid surface) and a hydrogel as countersurface, yielding a migrating hydrogel-solid contact. Adapted from Ref. [32], Copyright 2018 with permission from ACS Publications. (e) Hydrogel-hydrogel contacts in (1) unidirectional sliding of a ring-on disc, (2) eight flat pins-on-disc, (3) four flat pins-on-disc and (4) reciprocating sliding of a flat pin-on-disc [28]. Reproduced with permission from Simič, R. et al., Importance of Hydration and Surface Structure for Friction of Acrylamide Hydrogels. Tribology Letters, 2020, 68, 1-12. In all cases, the hydrogels were completely immersed in water.

The goal of this review was not to cover all of the literature on hydrogel lubrication but to discuss specific models that have attempted to quantify hydrogel friction. More specifically, this review is mainly focused on adhesive contacts between a solid surface and a hydrogel, although some findings obtained on repulsive contacts are presented to explain available models for viscous dissipation. The paper is organized in three main sections. First, the main models for hydrogel friction are described. One subset of models takes a polymer physics approach based on the scaling laws proposed by de Gennes [13], and emphasizes the role of the mesh size both in boundary and in hydrodynamic lubrication. In contrast to this, the second type of models emphasizes the role of a thin fluid film effectively separating the countersurfaces at low velocities and/or full fluid film lubrication at fast sliding velocities. This paper discusses these apparently contradictory models for single network hydrogels and their conditions of validity. This is followed by a detailed description of our own 
model for hydrogel friction and its application to two different hydrogels: polyacrylamide and agarose hydrogels, which lead to adhesive and repulsive contacts with a solid surface, respectively. We conclude with closing remarks and opportunities for future research to the best of the authors' knowledge.

\section{Hydration Lubrication}

The brainchild of Klein [33], the concept of a thin film of water of a few nanometers in thickness separating two surfaces decorated with polymer brushes found a way to explain lubrication mediated by synthetic and biological hydrogels and the low coefficients of friction $[15,20,34,35]$. Imagine the hydrated polymer chains at the hydrogel's surface sliding past each other. The so-termed hydration lubrication presumes the absence of contact between the polymer-bearing surfaces as a result of repulsive interactions, mainly elastic and osmotic in origin, although electrostatic interactions also contribute in the case of charged hydrogels [36]. Hence, it attributes the low friction coefficients to the small viscous force upon shear of a thin (hydration) film separating the surfaces [37-39]. Please note that this model considers that the velocity is not high enough for a hydrodynamic lift to happen. As originally postulated by Klein, this mechanism is based on the fast relaxation dynamics of the water molecules in the hydration shell of the charged monomers despite the applied pressure and the confined geometry (Figure 3a,b). The small energy dissipation upon shear relies on the property of confined water to preserve its bulk fluid behavior under confinement. This is in contrast to other solvents that undergo a dramatic increase in viscosity under confinement. This concept was extended to neutral brushes based on molecular dynamics (MD) simulations (Figure 3c) [40,41] and normal force and nanotribological measurements with a Surface Forces Apparatus [42,43], and to hydrogels coinciding with the discovery of a brush-like superficial region [44]. However, MD simulations of the tribological performance of neutral polymer (dextran) brushes against a hard wall recently showed a good agreement with experiments only after consideration of an effective polymer-wall attraction that leads to intermittent contact between polymer chains and wall [45]. The direct contact between the polymer and a solid surface has also been observed either directly via imaging [46,47] or indirectly through the measured stick-slip [23,24]. This reminds us that the existence of a thin film is not universal, but strongly depends on the chemical interactions between the polymer and the solid countersurface.
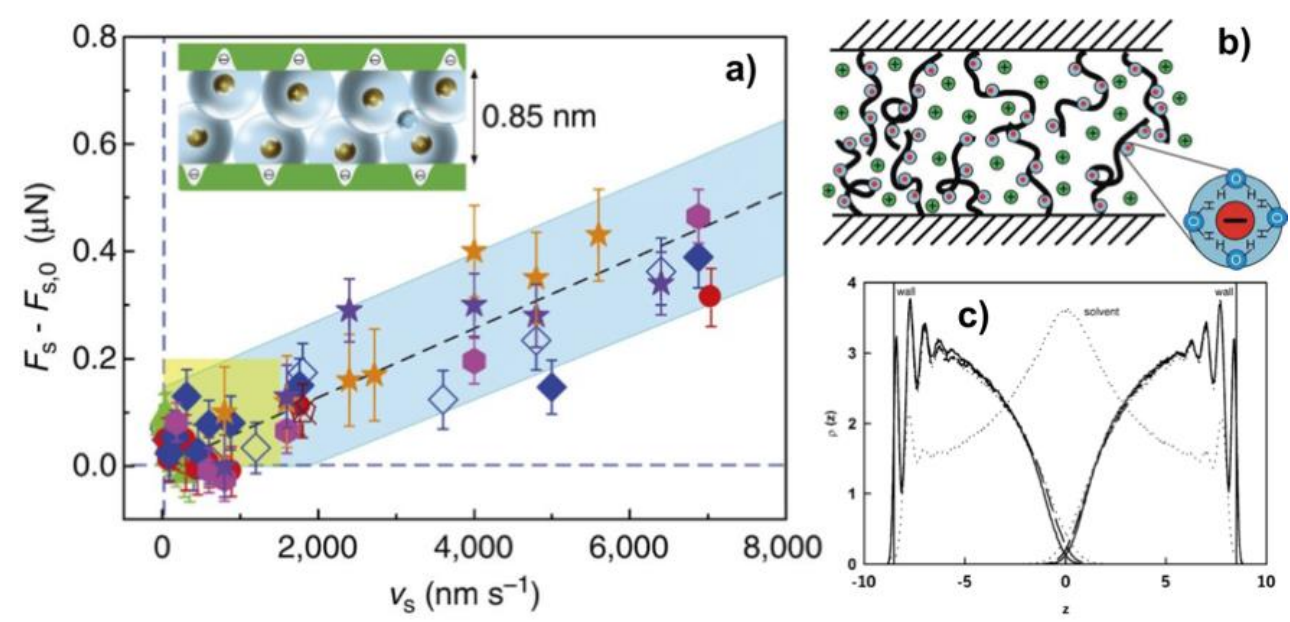

Figure 3. Origins of hydration lubrication. (a) The linear variation of the shear force $F_{\mathrm{S}}$ with the sliding velocity $\left(v_{\mathrm{S}}\right)$ characterizes the viscous dissipation via a Newtonian fluid. In this example, ions with their hydration shells are confined between mica surfaces [48]. The inset is approximately to scale and illustrates the filling of the intersurface gap by the hydration water. Different symbols are from different measurements. Full and empty symbols correspond to increasing and decreasing $v_{\mathrm{s}}$, respectively. From the slope of the blue band an effective viscosity for the confined film $\eta_{\text {eff }}=0.22 \pm 0.07 \mathrm{~Pa} \cdot \mathrm{s}$ is obtained, which is $\sim 250$ times larger than the viscosity of water. This increase does not result from the confinement of water (since the viscosity remains equal to the bulk viscosity [49]) but instead the higher 
viscous dissipation of the hydration water is associated with the primary hydration shell surrounding the ion. Reprinted by permission from Springer Nature Customer Service Centre GmbH: Nature Research, Nature Communications, Origins of hydration lubrication, Ma, L. et al., (2015). (b) Cartoon representing polyelectrolyte brushes with a hydration shell. Hydration lubrication is a general mechanism that can be relevant whenever contacting hydrated species are in relative motion, like also in hydrogels. Reproduced with permission from Ref. [32]. Copyright 2013 Springer. (c) MD simulations of the shear between neutral polymer brushes show the increased density of solvent between the polymer brushes, providing a film of low viscosity that is responsible for the decrease in friction. Reproduced from Ref. [41] with permission from the PCCP owner societies.

\section{Elastic Contribution to Frictional Dissipation}

Gong's studies of hydrogel lubrication significantly contributed to advancing the knowledge of mechanisms underlying hydrogel friction $[20-22,27,50]$. The main idea is that friction results from two major contributions, the energy dissipated due to the rupture of adhesive bonds across the interface and the viscous dissipation originated by the shear of the solvent film separating the two surfaces at sufficiently high sliding velocities [20]. Gong's model for adhesive contacts [27] assumes an intermittent and unconcerted adsorption and desorption of polymer chains to the solid surface. As the countersurface moves, the pinned polymer strands stretch, thereby storing an elastic force, $F_{e l}$ :

$$
F_{e l}=k_{B} T \cdot \frac{V \cdot t}{\xi^{2}}
$$

where $V$ is the sliding velocity, $t$ the contact time, and $k_{B} T$ the thermal energy per polymer chain. When detachment occurs, the stored elastic energy is dissipated. The elastic contribution to hydrogel friction depends on the relation between contact time $(t)$ and the relaxation dynamics, which are characterized by the lifetime of the adsorbed chain $(\bar{t})$ and the polymer relaxation time $\left(\tau_{A}\right)$. At the interface, $\tau_{A}$ represents the time for re-attachment of the polymer after detachment. The model predicts an increase in friction with sliding velocity due to the increasing number of adhesive bonds-often labelled as velocity-strengthening friction. Because the lifetime decreases with an increase in velocity, the adhesive frictional force exhibits a decreasing trend at sufficiently high velocities—or velocity-weakening regime-and a peak or plateau in between (Figure 4). Gong observed that weakly adhesive contacts showed a peak in friction at a velocity $\sim 2 \xi / \tau_{A}\left(v_{f}\right.$ in Figure 4) [51]. At sufficiently high sliding velocities, hydrodynamic lubrication was assumed to dominate over the contribution of the elastic friction.

Improvements of the adsorption-desorption model have been proposed, e.g., the so-called Population Balance equation (PBE) [52]. Instead of the Hookean spring model to calculate $F_{e l}$, the PBE model includes a finitely extensible non-linear elastic model for the stretching of the polymer. In addition, a viscous retardation stress accounts for the relative motion of the polymer chains surrounding the stretched polymer chain. Furthermore, it considers an average bond age $t_{a}$ that differs from its lifetime $\bar{t}$. Gupta et al. applied the PBE model to the friction of gelatin hydrogels [53]. The model was able to predict the increasing friction with gelatin concentration (and decrease in mesh size) originating from the enhanced adhesive bonds at the interface. Furthermore, the model was used to derive scaling laws of frictional parameters. An interesting result is that the shear modulus was observed to depend on the sliding velocity. This is an inherent property of a viscoelastic material, which behaves more fluid-like at low shear rates and more solid-like at higher shear rates. The work also showed that the Hookean approximation is valid for hydrogels, mainly because of the weak interactions at the interface. Nevertheless, if the binding interactions were to be strong (e.g., via electrostatic interactions), the polymer chains could be extended beyond the linear regime. 


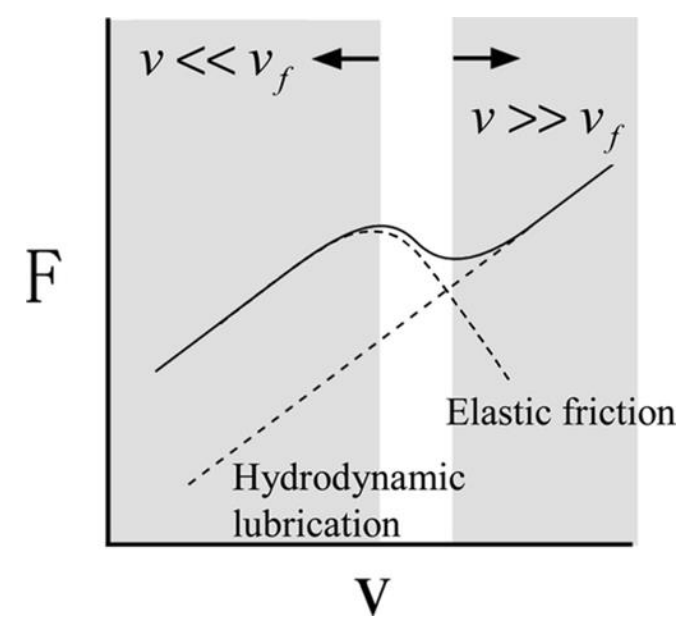

Figure 4. Schematics for the velocity dependence of the friction force at an adhesive contact between a hydrogel and a solid surface according to the elastic-hydrodynamic model. The model distinguishes three regimes: if the sliding velocity $V \ll v_{f}$, the frictional force is due to the elastic energy of stretched polymer chains dissipated upon rupture, and it increases with the sliding velocity. If the sliding velocity is high enough $\left(V>v_{f}\right)$ the polymer does not have enough time to adsorb to the solid surface, and the friction force decreases with increase in velocity. At very high sliding velocities $\left(V \gg v_{f}\right)$, the onset of hydrodynamic lubrication is proposed. Reproduced from Ref. [50], Copyright 2005 with permission from ACS publications.

An adhesive model was also proposed by Baumberger for gelatin-glass tribopairs [54,55]. As in Gong's model, the hydrogel surface consists of polymer blobs with a characteristic size close to $\xi$, and the blobs adhere to the glass surface due to van der Waals interactions, electrostatic attractions or hydrogen bonds. Baumberger's studies demonstrated the scaling of friction with the surface density of adhesive blobs, in marked contrast to the mechanism of hydration lubrication for repulsive contacts. This was further supported by observations of intermittent sliding or stick-slip due to the periodic attachment (detachment) of the network to (from) the countersurface, which was denoted as "self-healing slip pulses" [54]. Here, frictional slip occurs by propagating pulses that can self-heal (re-pin), by analogy to intermittent fault dynamics during an earthquake [56]. At velocities below a critical value, re-pinning occurs at the pulse's trailing edge and at once throughout the contact. The velocity of the slip or fracture front $\left(V_{\text {tip }}\right)$ was measured and found to depend on the mesh size and the collective diffusion coefficient of the polymer network $\left(D_{\text {col }}\right)$ as $V_{\text {tip }} \sim D_{\text {col }} / \xi$ [55]. Once the velocity exceeds a critical value, adhesive bond formation is insignificant, and the sliding becomes smooth. Here, the authors proposed that friction originates from the shear of a polymer solution film having a thickness comparable to that of the mesh size of the hydrogels and exhibiting shear thinning behavior; this is discussed later in detail. It is noteworthy that this work also introduced the concept of "aging", namely that the binding strength at the glass-hydrogel interface depends on the contact time.

\subsection{The Concept of Critical Velocity}

A common concept in Baumberger and Gong's works is the existence of a critical velocity at which the lubrication mechanism transitions to viscous dissipation. This critical velocity has been related to the dimensionless Weissenberg number $\left(W_{e}\right)$, which is classically used to describe viscoelastic flow. The Weissenberg number compares elastic and viscous forces via the ratio between a relaxation time and an experimental time $\left(\tau_{A} / t\right)$. If the relaxation time of the polymer $\left(\tau_{A}\right)$ is equal to the interaction time with the counter-surface $(t)$, elastic and viscous forces are of similar relevance. This happens at a critical velocity $V_{c}$, where $V_{c} \sim \xi / \tau_{A}[27,50]$. If $V<V_{c}$, elastic forces are more relevant than viscous forces ( $t>\tau_{A}$, low $W_{e}$-numbers), and vice versa if $V>V_{c}$. 
Table 2 shows the critical velocity $V_{c}$ that we calculated for the experiments in previous works. The magnitude of the transition velocity $\left(V_{\text {exp }}^{*}\right)$ was extracted from the reported data, considering that viscous dissipation happens at the highest sliding velocities. As Table 2 demonstrates, $V_{\exp }^{*}$ is various orders of magnitude smaller than the calculated critical velocity, $V_{c}$. It is intuitive that polymer chains are free to fluctuate at hydrogel-hydrogel interfaces. However, under the confinement provided by a solid surface in an adhesive contact, the polymer relaxation in the near-surface region should be restricted to some extent when water is drained, as reported for polymer brushes [38]. In addition, the critical velocity was estimated with the mesh size of the bulk hydrogel $\xi$. However, recent studies have demonstrated that the near-surface region of hydrogels may have structural characteristics that differ from the bulk as a result of the modified polymerization reaction close to the interface. All this is expected to contribute to the deviation between $V_{\text {exp }}^{*}$ and $V_{c}$ shown in Table 2.

Table 2. Calculated critical velocity $\left(V_{c} \sim \xi / \tau_{A}\right)$ and observed transition velocity in experiments $\left(V^{*}\right)$. Calculations at $298 \mathrm{~K}$ and for a solvent viscosity of $0.89 \mathrm{mPa} \cdot \mathrm{s}$. Single-network hydrogels, unless indicated. The mesh size was measured in Ref. [57] using Small Angle X-Ray Scattering and in Refs. [23,50,55] using Dynamic Light Scattering. * The abbreviation PAMPS stands for poly(2-acrylamido-2-methylpropanesulfonic acid).

\begin{tabular}{ccccc}
\hline Hydrogel & $\xi(\mathrm{nm})$ & $V_{c}=\frac{\xi}{\tau_{A}}=\frac{k_{B} T}{\eta \xi^{2}}(\mathrm{~m} / \mathrm{s})$ & $V_{\text {exp }}^{*}(\mathrm{~m} / \mathrm{s})$ & Ref. \\
\hline PAMPS*-PAAm double network hydrogel & $0.7-77$ & $9.4-7.78 \times 10^{-4}$ & $<0.03($ not always observed) & {$[50]$} \\
Porcine skin gelatin & $6.8-12$ & $0.09-0.03$ & $9 \times 10^{-5}-3.5 \times 10^{-4}$ & {$[55]$} \\
polyacrylamide & $1.3-9.4$ & $2.7-0.05$ & $\sim 1 \times 10^{-3}$ & {$[57]$} \\
polyacrylamide & 7 & 0.094 & not always observed) & not observed \\
polyacrylamide & $7.1-9.9$ & $0.091-0.047$ & in the range $3 \times 10^{-6}-4.5 \times 10^{-3}$ & {$[58]$} \\
& & & $1 \times 10^{-3}-10^{-6}$ & {$[23,32]$} \\
\hline
\end{tabular}

\subsection{Relevance of Microstructural Gradients}

A synthesis-sensitive graded microstructure has been reported for polyacrylamide hydrogels $[27,44,59]$. The hydrogel's surface, when molded against a hydrophobic surface, results in a soft, highly hydrated and loosely crosslinked ("brush-like") near-surface region (Figure 5a). A brush is characterized by its height $\left(\xi_{s}\right)$ [60]. The relaxation of dangling chains differs from the relaxation of the crosslinked polymer network, which diffuses in a cooperative motion. This brush-like region can have a thickness as large as $\sim 4 \mu \mathrm{m}$ [44]. For example, Shoaib et al. found that PAAm hydrogels with 4,6 and $9 \mathrm{wt} \%$ monomer and $0.1,0.3$ and $0.45 \mathrm{wt} \%$ crosslinker, when molded against hydrophobic glass slides treated with dichloro dimethylsilane, have a loosely crosslinked surface layer with an approximate thickness of $\sim 640,330$ and $250 \mathrm{~nm}$, respectively [32]. We discuss results for these hydrogels later.

The influence of the brushy surface region on the friction coefficient is remarkable (Figure $5 b$ ). Its presence reduces friction significantly. It can also be expected that the existence of this surface layer affects $V_{c}$. In fact, the fits of the viscous-adhesive model to the measured friction force between PAAm hydrogels and a silica sphere [23] lead to relaxation times for attachment/detachment of the polymer to/from the countersurface that are much longer than expected for the cooperative diffusion of blobs of size $\xi$. This might be caused by the contribution of the characteristic length of the near-surface region, $\xi_{s}$, instead of the mesh size of the bulk gel. Halperin [61] estimated the longest relaxation time required for a tethered polymer chain to stretch or collapse a distance equal to its thickness by the reptation time, $\tau_{\xi_{s}}=\eta_{s} \xi_{s}^{3} / k_{B} T$. Table 3 shows that the relaxation times estimated for $\xi$ and $\xi_{s}$ for three different polyacrylamide hydrogels differ by about $4-5$ orders of magnitude, with $\tau_{\xi} \ll \tau_{\xi_{s}}$. Accordingly, very different relaxation dynamics can be expected for the polymer at the interface. Please note that this estimation neglects that the applied pressure can yield the drainage of water and an increase in polymer concentration, as well as that the polymer chains can be crosslinked on both ends, all of which can further slow down the polymer dynamics. 
a) hydrophilic glass-molded hydrogel surface

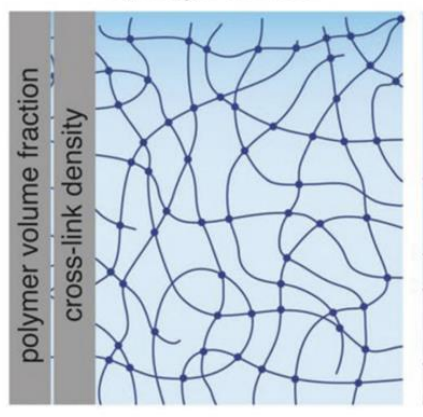

hydrophobic PDMS-molded hydrogel surface

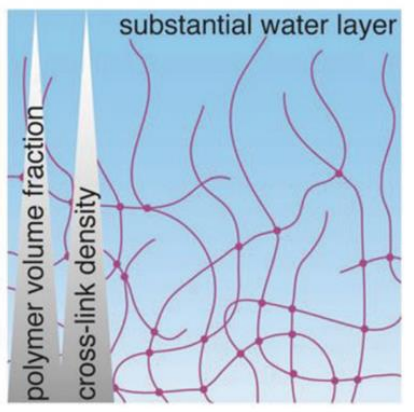

b)

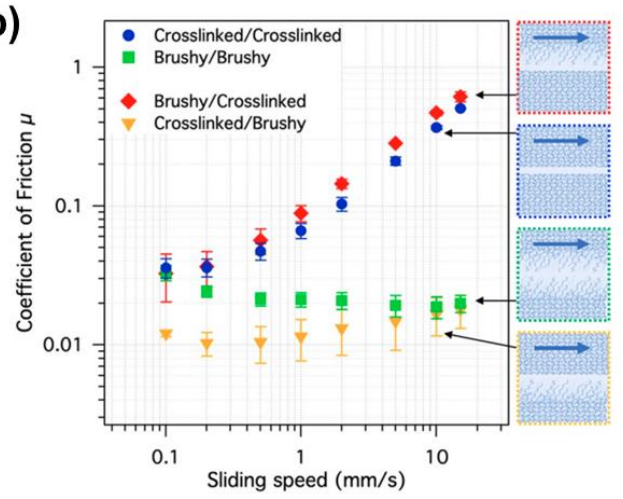

Figure 5. Influence of microstructural gradients on hydrogel friction. (a) Schematics representing the different microstructure of hydrogel surfaces when molded against a hydrophilic and a hydrophobic surface, respectively. The former yields a uniform (gradeless) polymer network, whereas the latter leads to a brush-like surface region that exhibits a lower polymer volume fraction and a reduced crosslink density compared to the bulk hydrogel [44]. Reprinted from Gombert, Y. et al. Structuring Hydrogel Surfaces for Tribology. Advanced Materials Interfaces, 2019, 6, 1901320. Copyright (2019) via Creative Commons license: https://creativecommons.org/licenses/by-nc/4.0/. (b) Change of the friction coefficient for different hydrogel-hydrogel contacts under a pressure of $6 \mathrm{kPa}$. Lower friction coefficients with weak velocity dependence are obtained for brush-brush configurations and for migrating brush-crosslinked network contacts. Higher friction coefficients that increase with sliding velocity are measured for contacts between crosslinked networks and for static brush-crosslinked network contacts. The measurements in (b) were carried out using a microtribometer in a flat pin-on-disc configuration in reciprocating motion (configuration 4 in Figure 2e) [28]. Reprinted from Simič, R. et al. Importance of Hydration and Surface Structure for Friction of Acrylamide Hydrogels. Tribology Letters, 2020, 68, 1-12.

Table 3. Comparison of the relaxation times $\left(\tau_{A}\right)$ estimated using the bulk mesh size $\xi$ and the length of the brushy region at the surface of the hydrogel $\xi_{s}$ for the polyacrylamide hydrogels investigated in Ref. [23]. The hydrogels were prepared with 4,6 and 9 wt $\%$ monomer and $0.1,0.3$ and 0.45 wt $\%$ crosslinker concentrations, and are labeled as PAAm- $4 \%$, PAAm- $6 \%$ and PAAm- $9 \%$, respectively. Calculations performed at a temperature of $298 \mathrm{~K}$ and for a solvent viscosity of $0.89 \mathrm{mPa} \cdot \mathrm{s}$.

\begin{tabular}{ccccc}
\hline & $\begin{array}{c}\xi \\
(\mathbf{n m})\end{array}$ & $\begin{array}{c}\xi_{\boldsymbol{s}} \\
\mathbf{( n m )}\end{array}$ & $\begin{array}{c}\boldsymbol{\tau}_{\boldsymbol{\xi}} \\
(\mathbf{m s})\end{array}$ & $\begin{array}{c}\boldsymbol{\tau}_{\xi \boldsymbol{s}} \\
\mathbf{( m s )}\end{array}$ \\
\hline PAAm-4\% & 9.9 & 640 & $2.1 \times 10^{-4}$ & 56.8 \\
PAAm-6\% & 8.2 & 330 & $1.2 \times 10^{-4}$ & 7.78 \\
PAAm-9\% & 7.1 & 250 & $7.8 \times 10^{-5}$ & 3.38 \\
\hline
\end{tabular}

\section{Poroelastically Induced Drainage of Water and Induced Frictional Dissipation}

Pressure-driven fluid flow in the hydrogel occurs only when the applied pressure exceeds the osmotic pressure of the hydrogel, $\Pi$ [62], and can also be the origin of frictional dissipation. The drainage of water upon indentation of the hydrogel with rigid probes has been described within the framework of poroelastic theories, which couples the elasticity of the gel network with the pressure-induced flow of water. The footprint of a poroelastically induced dissipation is a decrease in friction with an increase in sliding velocity, as the time for fluid drainage and the dissipation are reduced. This is often described in terms of a Peclet number (Pe), which gives the relation between diffusive and advective time, $\mathrm{Pe}=\tau_{W} V / a$, where $\tau_{W}$ is the poroelastic relaxation time, $V$ the sliding velocity, and $a$ the contact radius. The value of $\tau_{W}$ can be roughly estimated with the hydrogel diffusivity (Table 1), and depends on the mesh size, the elastic modulus of the hydrogel, and the applied pressure. Large Peclet numbers (large $\tau_{W}$ and fast sliding velocities) are thus associated with lower friction coefficients. 
Several analytical models have been derived for the poroelastically induced frictional dissipation [58,63,64]. Interestingly, Delavoipière et al. [62] identified two different regimes by visualizing the contact area during sliding through reflection interference contrast microscopy. In the low-velocity regime (i.e., when $\mathrm{Pe}<1)$, the contact line remains circular $(\zeta \sim 1)$ with a constant radius close to the radius achieved under static indentation loading at the same normal force $\left(a_{0}\right)$. Here, friction increases with sliding velocity (Figure 6). In the high-velocity regime ( $\mathrm{Pe}>1$ ), the contact radius decreases progressively with increasing sliding velocity while the asymmetry parameter $\zeta$ first decreases and then increases. This change in the shape of the contact area was demonstrated not to result from a hydrodynamic lift but from a pressure imbalance between leading and trailing edges arising from the poroelastically driven fluid flow, resulting in a lifting force. Most of the frictional dissipation was accounted for by a fracture mechanics approach, where the leading and trailing edges of the contact were viewed as closing and opening cracks, respectively, and dissipation arose from the poroelastic flow. Instead of considering fracture mechanics, the main idea in Reale and Dunn's for their poroelastically driven lubrication model is that a decrease in velocity promotes dehydration of the contact, which yields an increase in adhesion, and thereby, in friction. Accordingly, they modeled the friction coefficient as a function of the Peclet number and the interfacial energy [58].
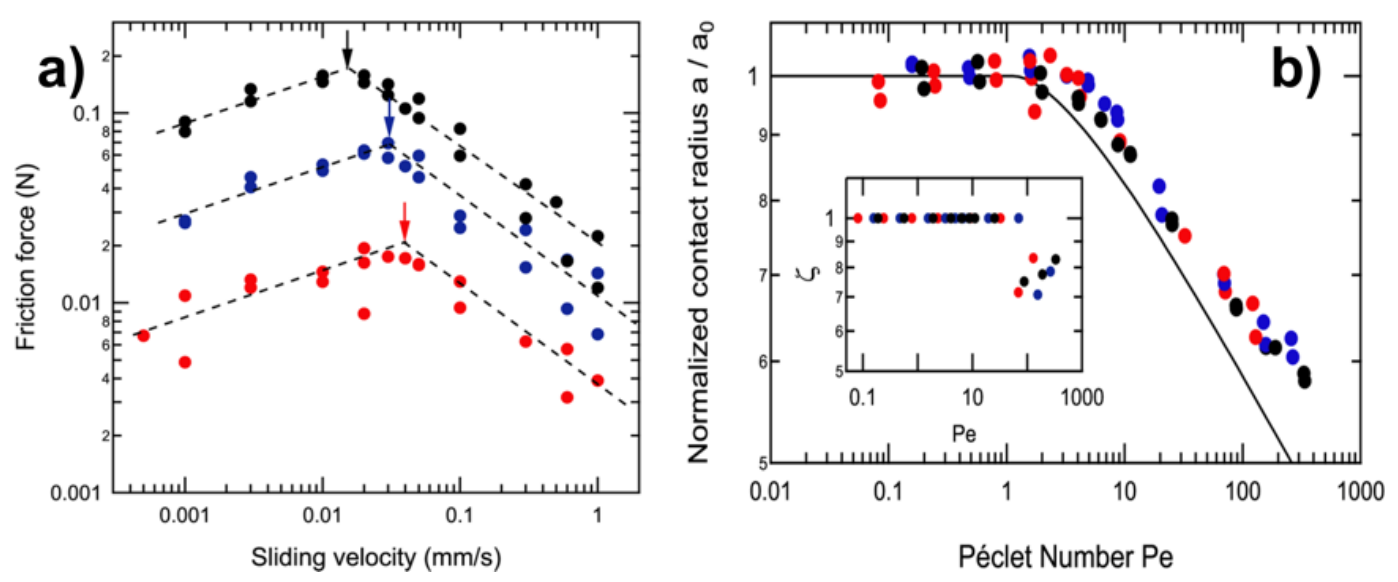

Figure 6. Decrease in friction and contact radius with increasing Peclet number due to the poroelastically driven flow in a hydrogel. (a) Friction as a function of sliding velocity for a thin poly(dimethylacrylamide) (PDMA) film coating a substrate fixed on a linear translation stage with a spherical glass probe as countersurface. The decrease in friction starts when the contact radius starts to decrease below that under static conditions, which happens when the Peclet number becomes larger than 1, as shown in (b). The inset in (b) shows changes in the asymmetry parameter $\zeta$ as a function of Pe. The black line in (b) corresponds to the prediction of the poroelastic model proposed in this work. Results at normal load of $50 \mathrm{mN}$ (red), $200 \mathrm{mN}$ (blue) and $600 \mathrm{mN}$ (black). (a,b) Reproduced from Ref. [64] Copyright 2018 with permission from ACS publications.

The findings by Delavoipière allow the identification of the experimental conditions at which poroelastically induced friction is of relevance. Table 4 shows the contact times $(t=a / V)$ as well as the poroelastic relaxations times, $\tau_{W}$, calculated with the tribological parameters provided in several reported experiments.

Table 4 shows multiple studies in which poroelastic drainage is of relevance. This is, of course, largely dependent upon the experimental conditions such as the sliding velocities and the contact pressures. In works by Reale and Dunn [58] and Delavoipière [64], poroelastic drainage is relevant under many experimental conditions, excluding the highest speeds probed (highest Pe numbers). In Ref. [23], the poroelastic drainage in PAAm- $4 \%$, PAAm- $6 \%$ and PAAm- $9 \%$ hydrogels is relevant, which mainly results from the small contact size in AFM experiments. 
Table 4. Comparisons of contact times $(t)$, poroelastic times $\left(\tau_{W}\right)$, Peclet numbers (Pe) and applied pressure $(P)$. The osmotic pressure, $\Pi$, of a polymer swollen in equilibrium with a good solvent is close to the shear storage modulus, G [14], and was measured only in some works. The data include polyacrylamide hydrogels with different monomer (acrylamide, AAm) and crosslinker (bisacrylamide, bis-AAm) concentrations from Ref. [23], PDMA hydrogels from Ref. [64] and adult bovine cartilage from Ref. [63].

\begin{tabular}{|c|c|c|c|c|c|c|}
\hline Ref. & Composition & $\begin{array}{l}t=a / V \\
(\mathrm{~s})\end{array}$ & $\begin{array}{l}\tau_{W} \\
(\mathrm{~s})\end{array}$ & $\begin{array}{c}P \\
(\mathbf{k P a})\end{array}$ & $\underset{(\mathrm{kPa})}{\Pi}$ & $\begin{array}{l}\mathrm{Pe} \\
(-)\end{array}$ \\
\hline Moore [63] & $\begin{array}{c}\text { Adult } \\
\text { bovine } \\
\text { cartilage }\end{array}$ & $0.09-9.5$ & - & $337-70$ & - & - \\
\hline Reale [58] & $\begin{array}{c}\text { AAm- } 10 \%, \\
\text { bis-AAm- } 0.6 \%\end{array}$ & $0.03-113$ & 34.9-294 & 5.0-10 & $\sim 8$ [65] & $3.5-5500$ \\
\hline Delviapore [64] & PDMA & $\begin{array}{c}\sim 0.007-120 \\
\sim 0.13-200\end{array}$ & $\begin{array}{c}9.1 \\
39.8\end{array}$ & - & - & $0.1-400$ \\
\hline Shoaib [23] & $\begin{array}{c}\text { AAm- } 4 \%, \\
\text { bis-AAm- } 0.1 \%\end{array}$ & $\begin{array}{l}0.009-9.78 \\
0.014-14.9\end{array}$ & $\begin{array}{l}39.7 \\
15.7\end{array}$ & $\begin{array}{c}0.4 \\
0.76\end{array}$ & $0.14 \pm 0.01$ & $\begin{array}{l}4-4000 \\
1-1000\end{array}$ \\
\hline Shoaib [23] & $\begin{array}{c}\text { AAm-6\%, } \\
\text { bis-AAm- } 0.3 \%\end{array}$ & $\begin{array}{c}0.0077-7.76 \\
0.009-9.83\end{array}$ & $\begin{array}{c}36.4 \\
9.8\end{array}$ & $\begin{array}{l}1.71 \\
2.58\end{array}$ & $\begin{array}{c}4.7-4700 \\
0.27 \pm 0.016\end{array}$ & $1-1000$ \\
\hline Shoaib [23] & $\begin{array}{c}\text { AAm-9\%, } \\
\text { bis-AAm- } 0.48 \%\end{array}$ & $\begin{array}{l}0.006-6.50 \\
0.007-7.72\end{array}$ & $\begin{array}{c}23.7 \\
4.8\end{array}$ & $\begin{array}{l}3.86 \\
5.28\end{array}$ & $\begin{array}{c}3.6-3600 \\
1.15 \pm 0.073\end{array}$ & $0.63-630$ \\
\hline
\end{tabular}

We also emphasize that the presence of microstructural gradients should affect the poroelastic relaxation. Depending on the stress distribution, the pressure-induced drainage of water can happen through the near-surface region with lesser resistance to flow in case of lower crosslinking degree, and/or through the bulk network. For instance, PAAm-6\% hydrogels have a surface characteristic length $\left(\xi_{s}\right)$ of $350 \mathrm{~nm}$ and a bulk mesh size $(\xi)$ of $\sim 8.2 \mathrm{~nm}$, and elastic moduli of 455 and $8700 \mathrm{~Pa}$, respectively. Upon indentation with a colloid $(\mathrm{R}=10 \mu \mathrm{m}, \mathrm{L}=10 \mathrm{nN})$, the corresponding poroelastic times are $\tau_{w}^{s}=1.23 \mathrm{~s}$ and $\tau_{w}=36 \mathrm{~s}$ for the surface and the bulk, respectively, hence resulting in an order of magnitude difference in poroelastic relaxation. Although a precise quantification is difficult because the stress field is unknown, it is important to consider that the poroelastic relaxation time may be significantly altered by the presence of microstructural gradients.

\section{Viscous Dissipation}

Hydrodynamic lubrication occurs when the sliding velocity is sufficiently high that there is a hydrodynamic lift, which yields a sustained fluid film separating the two surfaces in relative motion. The motion of a solid in a viscous fluid generates a shear stress in the fluid and brings it in motion. The viscous dissipation arises from the irreversible transformation of the work done by the shear in the fluid into thermal energy. With an increase in sliding velocity, the dissipated energy increases while this fluid film becomes thicker. Note that this deviates from the mechanism of hydration lubrication proposed by Klein and discussed earlier, which relies on the steric and osmotic repulsion provided by polymer brushes for the formation of a thin fluid film at the interface.

According to Newton's law of viscosity and taking into account de Gennes' scaling theory [66], Gong proposed the following expression for the friction force in the hydrodynamic regime:

$$
F_{v i s}=\eta_{s} A \frac{P}{c^{3 / 2}} V
$$

where $c$ is the polymer concentration, $P$ the applied pressure and $A$ the contact area between parallel plates with a relative sliding velocity $V$. Experimental results showed, however, a sublinear relation between $F_{v i s}$ and $V$,i.e., $F_{v i s} \sim V^{n}$ with $n \sim 0.21-0.55$ [20,51]. Hence, this expression was modified to account for the separation between the two surfaces and the Newtonian flow of the solvent within the gel, introducing the permeability $k$ of the hydrogel and its relation to the mesh size [20]. However, 
despite these improvements, the model underestimated the measured friction force by one order of magnitude. To justify this, the existence of bound water in the proximity of the polymer moieties and/or an increase in viscosity of the confined water was speculated [20]. This assumption is, however, doubtful, because the film thickness was estimated to be larger than $10 \mathrm{~nm}$ and an increase in the viscosity of water has not been demonstrated yet for such thick films. Furthermore, previous works have suggested that confined water retains fluidity under nanoconfinement [38].

Several experimental works have confirmed the sublinear relation $F_{v i s} \sim V^{n}$. For example, Kagata et al. [51] showed that the exponent $n$ increases from $\sim 0$ to $\sim 0.55$ with an increase in pressure, while the velocity dependence is less prominent (i.e., lower values of $n$ ) at slow sliding velocities. Without further proof, a non-Newtonian behavior of water and non-hydrodynamic dissipative mechanism were proposed to be responsible for the experimental results. A sublinear relation $F_{v i s} \sim V^{n}$ has also been reported for adhesive contacts above a transition or critical velocity $[23,51,54,67]$. Understanding the discrepancy among the reported exponents ( $n$ from $\sim 0.02$ to 0.7 ) and its load dependence is still the subject of research to date [20,23,28,35,51,67].

One of the shortcomings of Equation (2) is that it neglects the hydrodynamic variation of the film thickness with velocity arising from the deformation of the hydrogel. The soft hydrogel is deformed by the lubrication pressure generated in the thin film, leading to a net lift force that maintains the film $[29,64,68]$. For the case of an isoviscous-elastic contact, Hamrock and Dowson [69] proposed a viscous shearing force as $F_{v i s}=\eta_{s} \frac{V A}{h}$, where $h \sim V^{0.65}$, yielding $F_{v i s} \sim V^{0.35}$, which is qualitatively in better agreement with experimental results. Ureña et al. [67] showed, however, that the elastohydrodynamic theory underestimates the friction coefficient between two polyacrylamide hydrogel surfaces, and hence, a quantitative agreement is still lacking. The observed discrepancy could arise from neglecting the permeable nature of the hydrogels or from the geometrical differences between a ball-on-flat and a flat-on-flat contact, but a precise analysis has not been performed yet.

A transition from a low, speed-independent friction coefficient (at slow sliding speeds) to a speed-dependent friction coefficient $\mu_{v i s} \sim V^{0.5}$ was observed for Gemini hydrogel (repulsive) interfaces by Sawyer's group (Figure 7b) [57,70]. Although an exponent of 0.5 could be predicted by an elastohydrodynamic approach, the predicted friction coefficient was more than one order of magnitude lower than the measured values. The authors proposed "thermal-fluctuation lubrication" as the underlying mechanism at slow sliding velocities. That is, random thermal chain fluctuations-of length $\xi$-at the interface relax the shear stress generated during sliding and provide a blurred interface over which the barrier to sliding is effectively reduced compared to a hard interface. This is reminiscent of the polymer fluctuations shown in MD simulations of polymer brushes under shear [45]. The transition to higher friction coefficients at high sliding velocities was not attributed to full-fluid film (hydrodynamic) lubrication. Instead, it was proposed that the polymer relaxation was still involved in the lubrication of the repulsive contact (Figure 7a). However, the exact mechanism was not elucidated [31,57]. More recently, Simič et al. [28] proposed that the weak dependence of friction on velocity at slow sliding velocities in repulsive contacts reflects brush-like lubrication, i.e., hydration lubrication, and hence, it is dominated by the shear of the solvent.

A recent comprehensive study of the friction of polyacrylic acid (PAA), PAAm and agarose hydrogel spheres sliding on smooth solid surfaces yielding repulsive contacts has emphasized the relevance of hydrodynamics in hydrogel friction [29]. Based on the relation between the measured friction coefficient and the hydrogel mesh size, the friction force at low velocities is consistent with the dissipation by a hydrodynamic flow through the porous hydrogel network:

$$
F_{v i s}=\eta_{s} \frac{V A}{\xi}
$$

This differs from the brush-like hydration lubrication proposed by Spencer [28] or the thermal fluctuation lubrication suggested by Sawyer [57]. It is noteworthy, though, that the mesh size was not experimentally determined, but approximate values were assumed based on other works, and hence, 
perhaps only a qualitative comparison to the experimental results is granted here. At high velocities, a mesoscopic lubricating liquid film was presumed to form between the hydrogel and the solid surface. Agreement with elastohydrodynamic theory was only observed for the softest hydrogels, and the origin of the discrepancy for the stiffer gels was not elucidated. Interestingly, the frictional force decreased by an order of magnitude between these two regimes and displayed slow relaxation over several minutes (Figure 8). This was interpreted as an interfacial shear thinning of the polymer due to its alignment in the shear direction within a confined volume, which reduced friction before the onset of hydrodynamic lubrication. The observed time dependence of the friction force in this intermediate regime was justified by the effect of confinement on prolonging the timescale required for the polymer to explore the configurational space. Kim and Dunn modeled the shear stress in this regime using a thixotropic flow model to represent the time-dependent structural changes of the interface [71]; however, instead, they attributed the time-dependent frictional response to transient interfacial rehydration. Rehydration could not explain Cuccia et al. results [29], though.
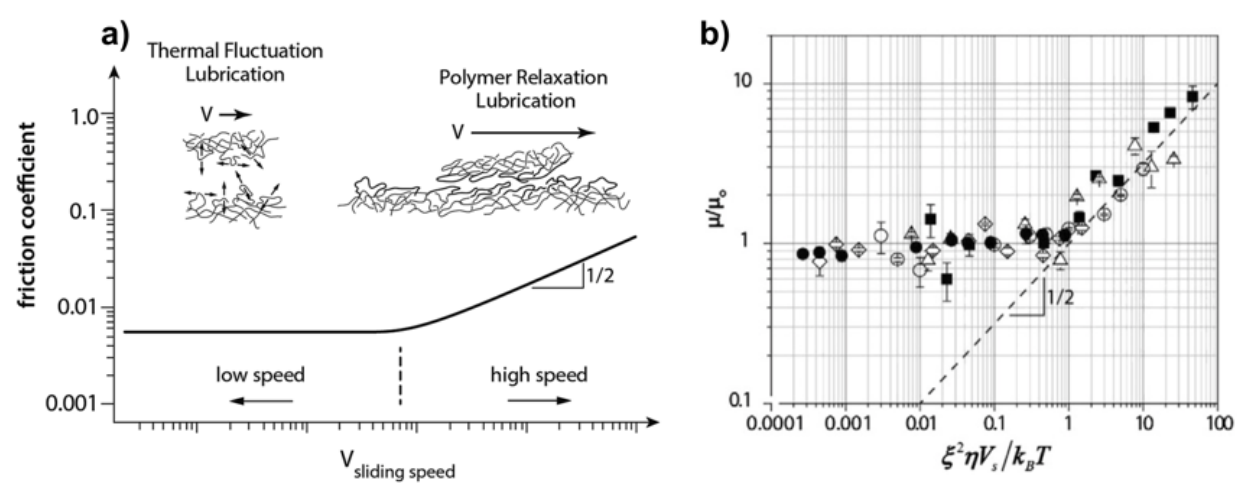

Figure 7. Gemini hydrogel lubrication model. (a) At low speeds, thermal fluctuation lubrication dominates, and the coefficient of friction is velocity-independent and small. At high speeds, polymer relaxation and adhesion are the dominant mechanism and the friction coefficient is higher and increases as $V^{0.5}$. Reproduced from Ref. [31] with permission from The Royal Society of Chemistry. (b) Collapsed experimental data on a master curve obtained by scaling the sliding velocity with $V_{c}$. Reproduced from Ref. [57] Copyright 2015 with permission from Elsevier B.V license number: 4901100446665.

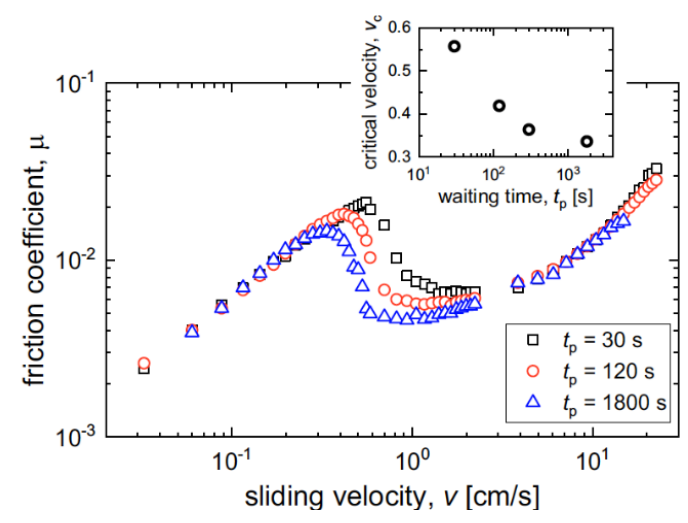

Figure 8. Friction as a function of sliding velocity for a commercial polyacrylic hydrogel particle sliding on a PAAm hydrogel surface. Three different values of the experimental running time are shown to illustrate the influence of time on the frictional response. Inset shows that the value of the critical velocity at which the peak in friction is observed decreases with increasing contact time [29]. The initial increase in friction with $\mathrm{V}$ is attributed to flow through the hydrogel, and the response at the highest sliding velocities is associated with full fluid film lubrication. Reprinted with permission from Cuccia, N.L. et al., Pore-size dependence and slow relaxation of hydrogel friction on smooth surfaces. PNAS, 2020, 117, 11247-11256. 
It is interesting that the time-dependent intermediate regime was only prominent in static contacts in Ref. [27]. Perhaps this is because the polymers near the hydrogel surface in a migrating contact only undergo shear loading transiently, which might hinder the shear-induced alignment and the confinement of entanglements compared to the situation in static contacts.

The idea of a non-hydrodynamic dissipative mechanism at high sliding velocities was elaborated by Baumberger et al. [55] for gelatin hydrogels and a glass countersurface yielding an adhesive interface. It was proposed that friction stems from the shear of a hydrogel film with a thickness of roughly the mesh size. The effective viscosity $\left(\eta_{e f f}\right)$ of this superficial region was defined as:

$$
\eta_{e f f}=\sigma \xi / V
$$

where $\dot{\gamma}=V / \xi$ is the shear rate and $\sigma$ the shear stress. Assuming that the behavior of the polymer chains follows that of the Rouse model [72], the relaxation was determined as $\tau_{R}=\frac{2 \eta_{s} \xi^{4}}{\pi b k_{B} T}$, where $b$ is the monomer size. The Rouse chain model assumes a dilute polymer solution below its point of entanglement, and hence, it deviates from the classical picture of a crosslinked network. Interestingly, it qualitatively agrees with the picture of a brush-like superficial layer for hydrogels with a graded microstructure (Figure 5a). The Weissenberg number $\left(W_{e}\right)$ was determined as the ratio between the stress relaxation time $\left(\tau_{R}\right)$ and an experimental time $\sim \xi / V$. Figure 9 shows how the experimental data corresponding to hydrogels with varying mesh size collapse onto a master curve $\eta_{e f f} / \eta_{s} \sim W_{e}^{-0.6}$. This indicates that the mesh size influences the viscous dissipation and that $\eta_{e f f} \sim \dot{\gamma}^{-0.6}$, thereby supporting the shear-thinning behavior of the superficial region of the gelatin hydrogels when sheared against a glass surface.

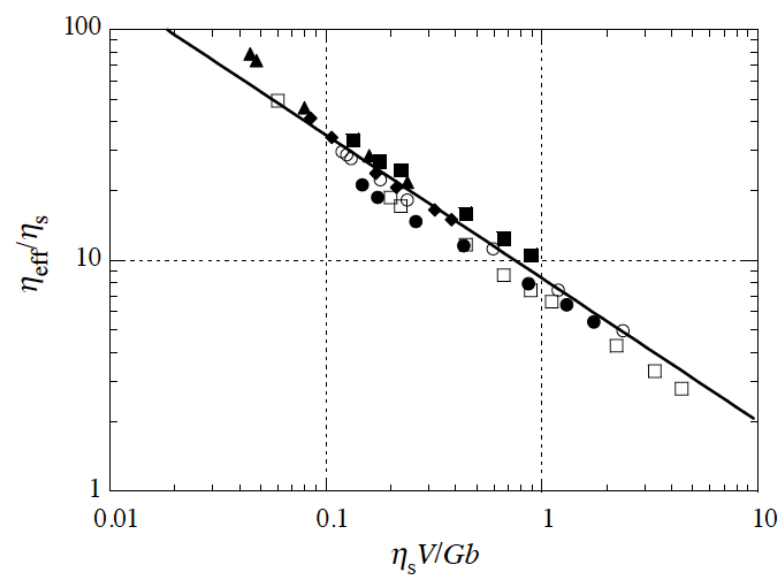

Figure 9. Relation between the reduced effective viscosity $\left(\eta_{e f f} / \eta_{s}\right)$ of the near-surface region of the hydrogel as a function of the Weissenberg number $\left(W_{e}=\tau_{R} V / \xi=\eta_{s} V / G b\right)$ for hydrogels of different compositions [55]. $G$ is the shear modulus of the hydrogel and $b$ is the monomer size. Reprinted with permission from Springer Nature Customer Service Centre GmbH: The European Physical Journal E, Self-healing slip pulses and the friction of gelatin gels, Baumberger, T., C. Caroli, and O. Ronsin Copyright (2003).

\section{The Viscous-Adhesive Model for Hydrogel Friction}

The viscous-adhesive model was introduced in Ref. [23] by the authors of this review to describe the frictional characteristics of the adhesive interface between polyacrylamide hydrogels and a silica microsphere. As in Schallamach's model for rubber friction [73] and in Gong's adsorption-desorption model, energy dissipation arises from the shear-induced rupture of the transient adhesive bonds (i.e., junctions) formed at the colloid-hydrogel interface. Our approach is conceptually similar to Schallamach's; however, the effect of the shear-assisted decrease on the energy barrier for bond rupture is neglected due to the small magnitude of the friction force. This enables us to derive a simple 
analytical expression for the adhesive (elastic, according to Gong's nomenclature) contribution to friction $\left(F_{a d h}\right) . F_{a d h}$ is thus given by the strain of the junction $(V \cdot t / d)$ multiplied by its shear modulus $(G)$ and by the area of the adhesive junctions $\left(A_{b}\right)$ :

$$
F_{a d h}=\frac{A_{b} G V t}{d} \sim \frac{A_{b} G V \tau_{0}}{d} \frac{\left(1-\left(1+\frac{l^{*}}{V \tau_{0}}\right) \exp \left(-\frac{l^{*}}{V \tau_{0}}\right)\right)}{1-\exp \left(-\frac{l^{*}}{V \tau_{0}}\right)}
$$

with $t$ being the time elapsed since the zero-state stress, $V$ the sliding velocity, $d$ the length of the polymer junction, $\tau_{0}$ the bond rupture time, and $l^{*}$ the yield length (so $l^{*} / d$ is the yield strain). The yield strain considers that rupture can also happen at the yield length, a concept introduced by Drummond et al. [74] for surfactant monolayers. The area in adhesive or pinned state $\left(A_{b}\right)$ is estimated from $A_{b}=A_{V} \frac{\left\langle t_{b}\right\rangle}{\left\langle t_{b}\right\rangle+\tau_{f}}$, with $A_{V}$ being the migrating contact area $\left(A_{b} \leq A_{V}\right), \tau_{f}$ the characteristic time of bond formation and $\left\langle t_{b}\right\rangle$ the mean life time of the adhesive junction, $\left\langle t_{b}\right\rangle=\tau_{0}\left(1-\exp \left(-\frac{l^{*}}{V \tau_{0}}\right)\right)$, which accounts for the probability that a junction is in adhesive state. Equation (5) predicts that $F_{a d h}$ can increase and decrease with $V$ and a peak or plateau can be achieved in the intermediate regime. The adhesive contribution to friction depends on characteristics of the polymer network at the confined interface $\left(G, d, l^{*}, \tau_{0}\right.$ and $\left.\tau_{f}\right)$. The derivation of Equation (5) is described in detail in the SI of Ref. [23].

The sliding contact area $A_{V}$ takes into account the poroelastically induced deformation of the hydrogel in colloidal probe AFM experiments, and hence, it can be smaller than the static contact area $A_{0}$. In the limit of small deformations, a sliding contact radius $a_{V}$ is estimated from:

$$
a_{V}^{2} \sim R \delta_{V}=a_{0}^{2} \frac{\delta_{V}}{\delta_{0}}=a_{0}^{2} \frac{\delta^{\prime}\left(1-\exp \left(-t^{\prime}{ }_{V} / \tau_{W V}\right)\right)}{\delta^{\prime}\left(1-\exp \left(-t^{\prime}{ }_{0} / \tau_{W 0}\right)\right)}
$$

$\tau_{W}$ being the poroelastic relaxation time of the hydrogel with an effective diffusivity $D$. We distinguish between $\tau_{W V} \sim \frac{a_{V}^{2}}{D}$ and $\tau_{W 0} \sim \frac{a_{0}^{2}}{D}$ for migrating (subindex "V") and static contacts (subindex " 0 "), respectively; similarly, $\delta_{V}$ and $\delta_{0}$ are the indentation depths of the migrating and static contacts. $D$ is assumed to be the same for both and $t^{\prime}$ is the contact time $\left(t^{\prime}{ }_{0}=\frac{\delta_{0}}{\dot{\delta}_{0}}\right.$ and $t^{\prime}{ }_{V}=\frac{a_{V}}{V}$ of the static and migrating contact, respectively). The indentation depths, $\delta_{V}$ and $\delta_{0}$, are described via a Kelvin-Voigt model, appropriate for polyacrylamide hydrogels [75]. Linearization of this expression leads to:

$$
a_{V}^{2} \sim a_{0}^{2} \frac{\frac{t^{\prime} V}{\tau_{P V}}}{\frac{t_{0}^{\prime}}{\tau_{P 0}}}=a_{0}^{2} \frac{R \dot{\delta_{0}}}{V a_{V}}
$$

Rearranging the above expression, the velocity-dependent contact radius $a_{V}$ and contact area $A_{V}$ are given as:

$$
\begin{gathered}
a_{V} \sim\left(\frac{a_{0}^{2} \dot{\delta_{0}} R}{V}\right)^{\frac{1}{3}} \\
A_{V}=\pi\left(a_{V}^{2}+\delta_{V}^{2}\right)
\end{gathered}
$$

Several works have attempted to visualize the migrating contact area to obtain a more precise estimation $[29,64,76]$; see, e.g., Figure 6 . The work by Delavoipière visualized a decrease in contact area $\left(A_{V}<A_{0}\right)$ with velocity at Peclet numbers larger than 1 .

On the other hand, the resistance to the motion of a solid (the silica microsphere) in a fluid leads to viscous dissipation, which constitutes the viscous contribution to friction. Here, we use a general expression for the viscous drag,

$$
F_{v i s} \sim \eta_{e f f} V \Omega
$$


where $\eta_{e f f}$ is the effective viscosity of the fluid and $\Omega$ is a geometric factor. For a plane-plane geometry, $\Omega=\frac{A_{V}}{h}$, where $h$ is the thickness of the sheared film and can include the hydrogel; $h$ might also depend on the sliding velocity, but this is neglected here. For a sphere-plane geometry like the AFM geometry in Figure $2 \mathrm{~d}, \Omega=\frac{16}{5} \pi R \log \left(\frac{2 R}{h}\right)$ [77]. The WLF equation, which has worked well to describe the shear-thinning behavior $(n<1)$ of nanoconfined polymers [77], is used to model the effective viscosity, $\eta_{\text {eff }} \sim \eta_{0} \dot{\gamma}^{n}=\eta_{0}(V / h)^{n}$. In the case of a Newtonian fluid $(n=0), \eta_{\text {eff }}$ should be equal to the viscosity of the film $\eta_{0}$. If the polymer influences the viscous dissipation, then the viscosity $\eta_{0}$ will deviate from the solvent viscosity, $\eta_{s}$.

The kinetic friction is given by

$$
F_{k}=F_{o}+F_{a d h}+F_{v i s}
$$

where $F_{o}$ is a velocity-independent term, which was observed in experiments [23]. Our more recent results on agarose hydrogels suggest that it might be of viscous origin, as argued later.

The minimization of $F_{k}$ yields an expression for the transition velocity $V^{*}$ at which $F_{k}$ achieves a minimum in friction. The simplest form of $V^{*}$ is obtained for parallel plates:

$$
\frac{\partial F_{k}}{\partial V}=0 \rightarrow V^{*}=\left(\frac{G l^{* 2}}{2(1+n) \tau_{f} \eta_{0}}\right)^{\frac{1}{2+n}}<V_{c}=\frac{\xi}{\tau_{A}}=\frac{k T}{\eta_{s} \xi^{2}}
$$

Based on this model, the transition velocity $\left(V^{*}\right)$ depends on the elasticity of the transient junctions, $G$, the yield length $l^{*}$, the time for the formation of adhesive bonds at the confined interface $\tau_{f}$, and the viscosity parameters $n$ and $\eta_{0}$ of the rheological model. Hence, this expression demonstrates that $V^{*}$ arises from the competition between adhesive and viscous contributions to friction, like $V_{c}$. However, there are obvious differences between the expressions for $V^{*}$ and $V_{c}$. First, $V^{*}$. accounts for non-Newtonian behavior, if present. Second, $\tau_{f}$ can deviate from $\tau_{A}$, e.g., if the confinement provided by the solid surface slows down polymer dynamics. Third, it considers that rupture can happen above a yield length $l^{*}$, and hence, it is not only dictated by the fluctuation dynamics of the polymer. We find that the observed transition velocity in our experiments [23] is of the same order of magnitude as the calculated velocity $V^{*}$ (see Figure 10) and much smaller than $V_{c}$ (see Table 2). The discrepancy is mainly due to the prolonged relaxation time, i.e., $\tau_{f}>\tau_{A}$.

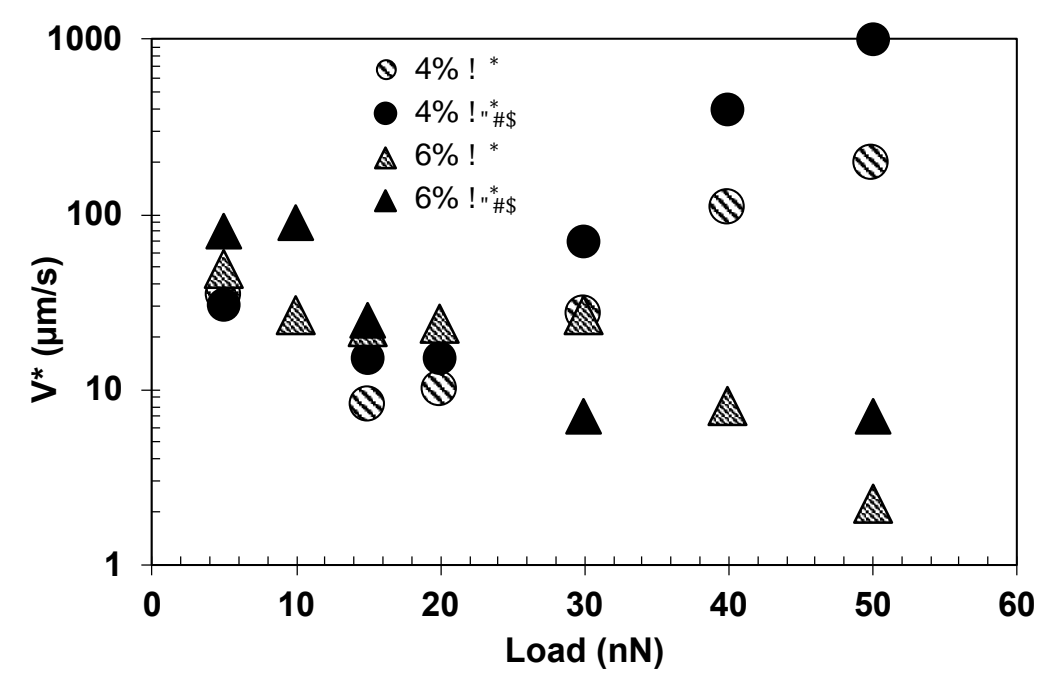

Figure 10. Comparison of the calculated transition velocity $V^{*}$ (Equation (8)) and of the measured transition velocity $V_{\text {exp }}^{*}$ in AFM friction-force experiments on polyacrylamide hydrogels with 4 and 6 wt \% acrylamide. Data used with permission from Ref. [23].

The viscous-adhesive model was used to describe the velocity and load-dependent friction force between a silica colloid and polyacrylamide hydrogels measured by AFM in Ref. [23]. 
Polyacrylamide can form hydrogen bonds with silica [78], and hence, the contact is adhesive. Here, we compare these results to the frictional response of agarose hydrogels, which exhibit a much weaker adhesion to the silica colloid. Agarose hydrogels were prepared by dissolving $1 \mathrm{wt} \%$ of agarose powder in DI water at a temperature of $80^{\circ} \mathrm{C}$ and continuous stirring at $375 \mathrm{rpm}$. After dissolution, $2 \mathrm{~mL}$ of the solution was pipetted into circular molds and left to gelate at room temperature for $30 \mathrm{~min}$. Then, DI water was added to the petri dishes, and gel samples were stored inside the fridge overnight. All gels were tested on the next day. The gel samples were rinsed with DI water to remove the uncrosslinked monomer. Polyacrylamide hydrogels were prepared with three different concentrations of the monomer, as reported in detail in our previous works [23,24,32]. All the measurements shown in this section were conducted with DI water as the solvent.

For the selected compositions of PAAm hydrogels, Table 5 shows the elastic moduli and the adhesion energy as measured by colloidal probe AFM, as well as the shear storage modulus as measured with a rheometer. The investigated agarose hydrogels exhibit an elastic modulus of $2.45 \pm 0.99 \mathrm{kPa}$ and zero adhesion energy under the same experimental conditions.

Table 5. Monomer and crosslinker concentration of three PAAm hydrogels, their corresponding elastic modulus and adhesion energy, measured by colloidal probe AFM indentation upon a load of $10 \mathrm{nN}$ and an indentation rate of $2 \mu \mathrm{m} / \mathrm{s}$. The shear storage modulus was measured with a rotational rheometer with a parallel plate geometry (DHR-3, TA instruments) as a function of frequency at a constant strain of $0.2 \%$.

\begin{tabular}{|c|c|c|c|c|}
\hline AAm (\%) & bis-Aam (\%) & Elastic Modulus (kPa) & Adhesion Energy $\left(\mathrm{J} / \mathrm{m}^{2}\right)$ & $\begin{array}{l}\text { Shear Storage } \\
\text { Modulus G' (Pa) }\end{array}$ \\
\hline $4 \%$ & 0.1 & $1.1 \pm 0.1$ & $7.98 \times 10^{-5}$ & $143 \pm 6.4$ \\
\hline $6 \%$ & 0.3 & $8.7 \pm 0.4$ & $3.25 \times 10^{-4}$ & $277 \pm 15$ \\
\hline $9 \%$ & 0.48 & $16.5 \pm 3.8$ & $5.52 \times 10^{-4}$ & $1151 \pm 73$ \\
\hline
\end{tabular}

Unknown parameters in Equations (5) and (6) are the thickness of the junction (d) and the thickness of the shear fluid film ( $h$ ), respectively. In Ref. [23], we assumed them to be equal to the indentation depth. Here, inspired by Baumberger's and Cuccia's works $[29,55]$, we assume that both $d$ and $h$ are close to the correlation length of the hydrogel in the near-surface region. However, this deviates from the mesh size of the bulk hydrogel $(\xi)$ due to the graded microstructure of our hydrogels. Hence, we assume here that both $d$ and $h$ are equal to the height of the brush-like surface region $\left(\sim \xi_{s}\right)$; see Table 3.

Fits of the viscous-adhesive model to the experimental results provide the magnitude of the fitting parameters. It is evident in Figure 11 that the friction force is higher for PAAm-9\% hydrogels. According to our model, this is mainly because the characteristic time for the formation of adhesive bonds $\left(\tau_{f}\right)$ is notably smaller for PAAm- $9 \%$ hydrogels compared to the other hydrogels, while the time for bond rupture $\left(\tau_{0}\right)$ is only marginally smaller than that of PAAm- $6 \%$ hydrogels (Figure 12a). Hence, the longer bond lifetime justifies the higher friction of PAAm- $9 \%$ hydrogels. Our model also reflects the effect of normal load on the polymer characteristics. Figure $12 \mathrm{~b}$ shows how the yield length of the polymer at the interface significantly increases with load for the three hydrogels, which contributes to the enhanced energy dissipation with load. In addition, an increase in load leads to a remarkable decrease in $\tau_{f}$, which implies that the formation of the adhesive bonds is facilitated with increasing load. This is likely because water is squeezed out and the polymer concentration in contact with the silica colloid increases. The change in $\tau_{0}$ with load is much more subtle, as inferred from the small size of the boxes in Figure 12a. The relaxation times of the polymer chains at the adhesive hydrogel-colloid contact are extended by several orders of magnitude compared to the unconfined polymer $\left(\tau_{f} \gg \tau_{A}\right)$. Please note that this has been proposed for polymer friction before [79]. This means that relaxation is slowed down and deviates from predictions of the scaling theory, likely due to the effect of confinement. This analysis thus provides insight into the relation between hydrogel microstructure and lubrication 
and shows that the confinement provided by the solid surface in an adhesive contact restricts the conformational entropy of the polymer and extends the relaxation time.
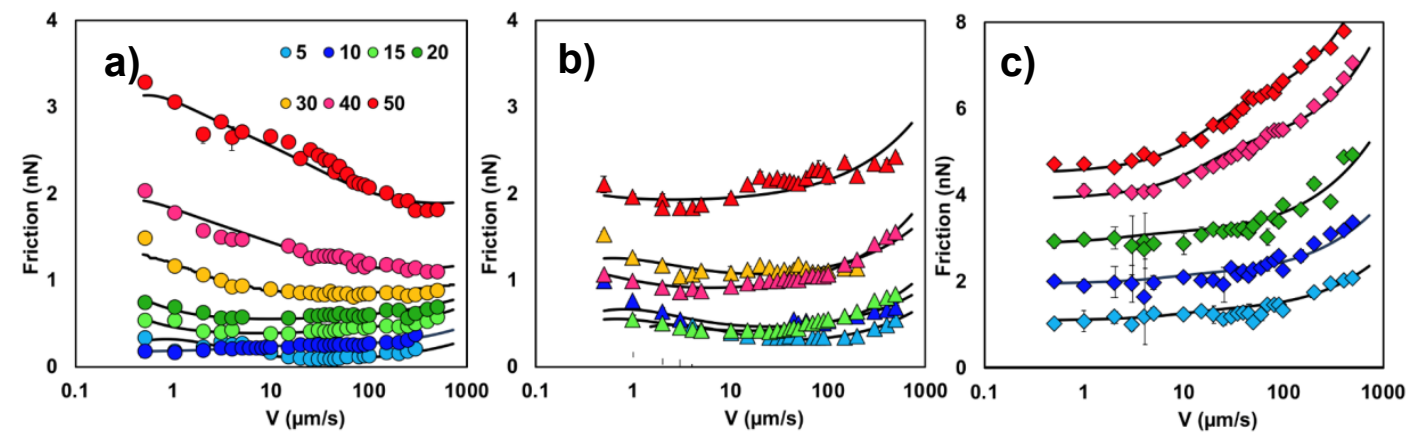

Figure 11. Friction force as a function of the sliding velocity for (a) $4 \%$ (circles), (b) $6 \%$ (triangles), and (c) $9 \%$ (diamonds) PAAm hydrogels at loads between 5 and $50 \mathrm{nN}$ (see legend in (a)). The black lines give the calculated friction force according to the viscous-adhesive model. Colloid diameter $=20 \mu \mathrm{m}$. Spring constant $=0.4 \mathrm{~N} / \mathrm{m}$. Note the different scales on the $Y$-axis of (c). Reprinted from Shoaib, $\mathrm{T}$. and R.M. Espinosa-Marzal, Insight into the Viscous and Adhesive Contributions to Hydrogel Friction. Tribology Letters, 2018, 66, 96.
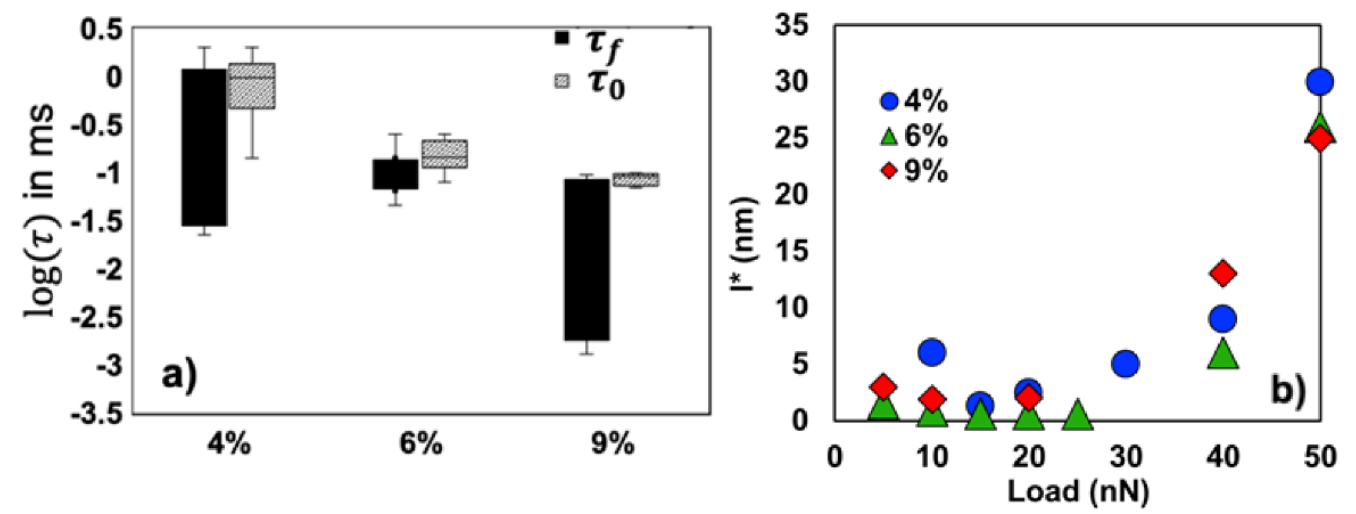

Figure 12. Parameters of the viscous-adhesive friction model for PAAm hydrogels (adhesive component). (a) Characteristics times for bond formation and rupture ( $\tau_{f}$ and $\tau_{0}$, respectively) in logarithmic axis, for loads between 5 and $50 \mathrm{nN}$, and (b) yield length $l^{*}$ of $4 \%-, 6 \%$ - and 9\%-PAAm hydrogels as a function of load. Data used with permission from Ref. [23]. The parameters shown here deviate from those in Ref. [23] because the results in this Figure were determined assuming that $h, d=\xi_{s}$ (see manuscript text).

As shown in Figure 11, the viscous contribution (i.e., the increase in friction with $V$ at high velocities) was most prominent for PAAm- $9 \%$ hydrogels in the range of investigated velocities. It should be noted that fits assuming a Newtonian behavior $(n=0)$ were possible $\left(\mathrm{R}^{2}>0.92\right)$, but a non-Newtonian behavior (shear thinning, $n=-0.32$ ) led to better fits, especially at the highest loads (Table 6). Please note that the Newtonian viscosity $\left(\eta_{0}\right)$ lies between 13 and $26 \mathrm{mPa} \cdot \mathrm{s}$, and hence, it is higher than that of water. We associate both the increase of Newtonian viscosity and the non-Newtonian behavior in the respective models with the influence of the polymer on the viscous dissipation. For PAAm- $6 \%$ hydrogels, the fits were slightly better assuming Newtonian behavior with a viscosity of 3-9 $\mathrm{mPa} \cdot \mathrm{s}$, which seems reasonable due to the smaller polymer concentration compared to PAAm- $9 \%$ hydrogels. Our current research is dedicated to providing evidence for shear thinning and a more precise rheological model. While we show here the results estimated with $h, d=\xi_{s}$, the calculations were also carried out with $\xi$, which led to similar conclusions, although the viscosity parameter $\eta_{0}$ was much higher in this case. 
Table 6. Fitting Parameters for $F_{v i s}$ for PAAm-6\%, PAAm-9\% and agarose hydrogels: Newtonian viscosity, $\eta_{0}$ with $n=0$, and viscosity parameter $\eta_{0}$ and exponent $n \neq 0$ for non-Newtonian behavior. The model considers the sphere-flat geometry with a factor $\Omega=\frac{16 \pi}{5} R \log \left(\frac{2 R}{\xi_{s}}\right)(R=10 \mu \mathrm{m})$.

\begin{tabular}{|c|c|c|c|c|c|c|}
\hline \multirow[b]{2}{*}{ Load $(\mathrm{nN})$} & \multicolumn{2}{|c|}{ PAAm $6 \%$} & \multicolumn{2}{|c|}{ PAAm 9\% } & \multicolumn{2}{|c|}{ Agarose 1 wt. $\%$} \\
\hline & $\begin{array}{c}\eta_{0} \\
(\mathbf{P a} \cdot \mathbf{s}) \\
(n=0)\end{array}$ & $\begin{array}{c}\eta_{0} \\
\mathbf{P a} \cdot \mathbf{s} / \mathbf{s}^{\mathbf{n}} \\
(n=-0.32)\end{array}$ & $\begin{array}{c}\eta_{0} \\
(\mathbf{P a} \cdot \mathbf{s}) \\
(n=0)\end{array}$ & $\begin{array}{c}\eta_{0} \\
\mathbf{P a} \cdot \mathbf{s} / \mathbf{s}^{\mathbf{n}} \\
(n=-0.32)\end{array}$ & $\begin{array}{c}\eta_{0} \\
(\mathbf{P a} \cdot \mathbf{s}) \\
(n=0)\end{array}$ & $\begin{array}{c}\eta_{0} \\
\mathbf{P a} \cdot \mathbf{s} / \mathbf{s}^{\mathrm{n}} \\
(n=-0.3)\end{array}$ \\
\hline 5 & 0.003 & 0.030 & 0.013 & 0.149 & & \\
\hline 10 & 0.003 & 0.032 & 0.018 & 0.213 & & \\
\hline 15 & 0.006 & 0.072 & & & 0.007 & 0.122 \\
\hline 20 & & & 0.026 & 0.314 & 0.008 & 0.141 \\
\hline 25 & 0.004 & 0.043 & & & 0.009 & 0.157 \\
\hline 30 & 0.007 & 0.081 & & & & \\
\hline 40 & 0.009 & 0.097 & - & 0.406 & 0.008 & 0.152 \\
\hline 50 & & & - & 0.443 & 0.008 & 0.145 \\
\hline
\end{tabular}

Friction force measurements on agarose hydrogels with a silica colloid are shown in Figure 13a. The friction force is quasi constant at slow velocities, and it increases remarkably with velocity above $\sim 10 \mu \mathrm{m} / \mathrm{s}$. The friction force at varying loads can be fit well by $F=F_{0}+\eta_{e f f} V \Omega$. Hence, consistent with the repulsive nature of the agarose hydrogel-colloid contact, the adhesive term $\left(F_{\text {adh }}\right)$ is zero. The fits to the experimental results assuming a Newtonian behavior ( $\left.\eta_{\text {eff }}=\eta_{0}=7-9 \mathrm{mPa} \cdot \mathrm{s}, n=0\right)$ are not satisfactory. In the range of investigated velocities, good fits are obtained by assuming a shear-thinning behavior, with an exponent $n \sim-0.3$. These results need to be considered with caution because the range of velocities accessible to our AFM is limited and it is possible that the viscous dissipation deviates at higher velocities. Although the available data are not sufficient to exclude either Newtonian or non-Newtonian behavior, they are sufficient to support the influence of the polymer on the viscous dissipation. Please note that a velocity-independent term $F_{0}$ is also needed here to reproduce the experimental results. Because the contact is repulsive, this term could be related to the hydration lubrication at slow velocities, which differs from our previous assumption [23]. Figure 13b shows the results for a 9\%-PAAm hydrogels for comparison; note the different scales on the $Y$-axis of both diagrams.
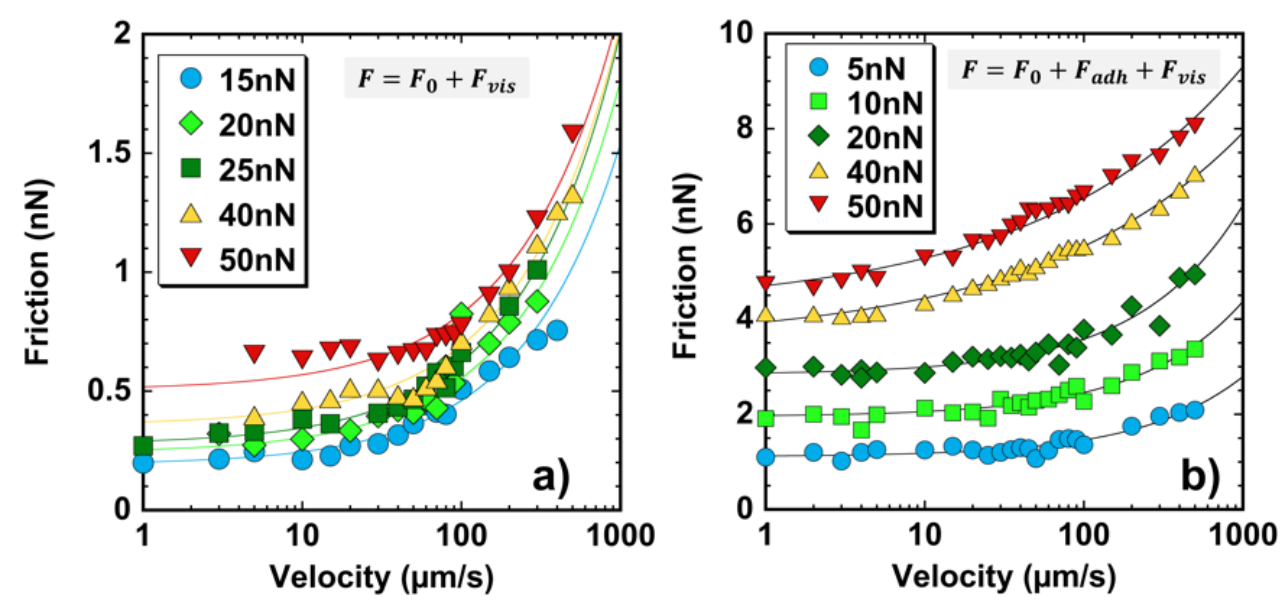

Figure 13. Fits of the viscous-adhesive model to the measured friction vs. sliding velocity for (a) agarose 1 wt \% hydrogels and (b) PAAm-9\% hydrogels. The viscous contribution presumes a shear-thinning model $(n=-0.32)$ for both systems. The agarose hydrogel forms a repulsive contact and hence the adhesive contribution to friction is zero. Data for Figure 13b used with permission from Ref. [23].

In summary, the viscous-adhesive model provides a theoretical approach to quantify frictional response of hydrogels by considering an interplay of adhesive and viscous dissipation directly arising 
from the hydrogel's microstructure. The model accounts for confinement effects, poroelastic deformation, and the influence of the polymer on the viscous dissipation in AFM friction force measurements. Limitations of this model and the experimental approach have also been highlighted, including the need for precisely quantifying the thickness of the sheared interfacial film, as well as elucidating the polymer's contribution to the viscous dissipation more precisely.

\section{Closing Remarks and Future Perspectives}

Although the models discussed here provide key physical insights into lubrication mechanisms, the above-outlined existing knowledge is only partial and qualitative, since it is still generally not possible to quantitatively predict the frictional characteristics of hydrogels based on their microstructure and tribological conditions. One of the goals of this revision was to present the richness and complexity of the mechanisms underlying hydrogel lubrication and friction. Part of the complexity arises from the experimental challenge posed by contact with a soft, permeable material with large concentrations of water. Opportunities for future research arise from the outlined knowledge gaps, some of which are emphasized in this section.

To date, it is still unclear under which tribological conditions a fluid film effectively separates permeable surfaces, like hydrogels, yielding hydrodynamic and hydration lubrication. This is mainly due to the inherent difficulty to experimentally prove the presence of this film and to determine its thickness. Recent neutron-reflectometry measurements by Spencer's group have demonstrated the presence of a thin film of water between hydrogels with a brush-like superficial layer and a silicon crystal upon an applied normal load [44]. This method could be theoretically extended to examine the change of film thickness with sliding velocity under tribological conditions for both adhesive and repulsive contacts. The major limitation of this technique is, however, its accessibility, as well as its lack of fine control of the force over a wide range of values.

As described earlier, assumptions for the viscous dissipation range from hydrodynamic lubrication mediated by the Newtonian behavior of the solvent to non-Newtonian shear of the hydrogel interfacial region and a polymer-relaxation lubrication mechanism. While all of these assumptions are consistent with experimental observations, measurements dedicated to examining the interfacial rheology of adhesive and repulsive contacts with hydrogels are still needed to provide fundamental insight into the interfacial behavior. Rheological models that specifically account for the time-dependent variation of friction in static contacts have been already considered [29,30,71]. The Surface Forces Apparatus (SFA) is a well-known method for precisely determining the thickness of (sub)nanometer fluid films, normal surface forces and friction. One advantage of this method is that the measurements of the film thickness are also possible during shear loading, so that it can determine the change in thickness with sliding velocity. Recent experiments on cartilage $[80,81]$ have demonstrated the capability of the SFA to investigate hydrogel-like materials, specifically their response to compression and steady shear. The SFA has been also used to investigate the nanorheology of polymer films [77,82,83], and we believe that extensions of the SFA could help understand the interfacial rheology of hydrogels under tribological conditions at the nanoscale, as well.

This review did not focus on the effects of chemical make-up and charge of hydrogel surfaces. Precedent works by Gong [20] and Sokoloff [15] have developed models for charged hydrogel friction. Between similarly charged surfaces, a fluid film can be expected [15]. Interestingly, it has been shown that friction can be controlled by adjusting the local molecular conformation of a polyelectrolyte brush via an alternating electric field [84]. The intensity of the applied field can regulate the stretching of the polymer chain while sliding, and thereby, the degree of interpenetration between opposite polymer brushes at the interface. The dynamics of the response is controlled by the relaxation times of the polyelectrolyte. While the molecular-level response to an electric field is relatively quick, less is known about the response dynamics of charged hydrogels. Electrotunable behavior offers opportunities for applications in soft robotics, among others, and hence, it is not only fundamentally interesting but also important for these applications. Furthermore, varying the fluid film properties through the 
modulation of an applied electric field or of the charge density of the hydrogel offers a new avenue to elucidate the mechanism of viscous and electroviscous dissipation.

The complexity of the lubrication mechanisms mediated by hydrogels also relies on other factors, including contact roughness and wear, not discussed in this review. Surface roughness can significantly affect the frictional characteristics. For example, while the friction at relatively smooth hydrogel surfaces follows well the elastic-hydrodynamic friction model by Gong [25], hydrogels with a surface roughness in the microscale $(1-10 \mu \mathrm{m})$ exhibit only a velocity-weakening regime. Similarly, the roughness of the hard surface is also shown to play a role in the frictional response below the critical velocity $[26,85]$. On the one hand, hydrogels have an inherent surface roughness owing to polymer dynamics at the interface. On the other, hydrogels with modulated surface topology can be prepared. Hence, it seems imperative to elucidate the influence of surface roughness on the lubrication mechanisms and friction models. Similarly, the relation between frictional dissipation and wear is still not well understood. While several works have examined the tribologically induced wear of hydrogels that can serve as biological replacement materials [19,86-88], the understanding of the mechanisms underlying tribologically promoted wear is not only lacking, but even more, the correlation is debated. Here, it is worth mentioning the work by Bonyadi et al. [89], who performed systematic measurements to correlate wear, friction and surface stiffness of polyacrylamide hydrogels. The study revealed a temporary stiffening of the surface layer upon wear, which undergoes osmotic re-swelling, regains the pristine surface stiffness and corresponding low friction. The authors attributed this behavior to the self-regenerating compliance and surface structure of hydrogels. Although more studies are needed to understand how a self-regeneration of chemically crosslinked hydrogels is possible, these results announce a new direction of research.

Another relevant phenomenon for the frictional dissipation of soft materials is static friction. Although only a handful of studies have focused on static friction of hydrogels [54,90-92], there is sufficient experimental evidence demonstrating the dependence of adhesion and static friction on contact time. While static friction is out of the scope of this review, it is worth mentioning that wear of soft biological materials has often been related to adhesion and static friction [93-95], and hence, future research should be dedicated to improving our understanding of the static friction of hydrogels, as well.

To conclude, hydrogels are undoubtedly of great interest to the tribology community, but there still remains much work to be done before we can design hydrogels for targeted tribological applications. Elucidating hydrogel lubrication mechanisms is not only paramount to understand better biolubrication, but also to advance the knowledge required to achieve this design goal.

Funding: The authors acknowledge funding support by NSF Grant No. 17-61696.

Acknowledgments: The authors acknowledge facilities provided by the Department of Civil and Environmental Engineering and Department of Materials Science and Engineering at the University of Illinois at Urbana-Champaign.

Conflicts of Interest: The authors declare no conflict of interest.

\section{References}

1. Dowson, D. Bio-tribology. Faraday Discuss 2012, 156, 9-30, discussion 87-103. [CrossRef]

2. Mantelli, F.; Argueso, P. Functions of ocular surface mucins in health and disease. Curr. Opin. Allergy Clin. Immunol. 2008, 8, 477-483. [CrossRef]

3. Sterner, O.; Karageorgaki, C.; Zurcher, M.; Zurcher, S.; Scales, C.W.; Fadli, Z.; Spencer, N.D.; Tosatti, S.G.P. Reducing Friction in the Eye: A Comparative Study of Lubrication by Surface-Anchored Synthetic and Natural Ocular Mucin Analogues. ACS Appl. Mater. Interfaces 2017, 9, 20150-20160. [CrossRef]

4. Swann, D.A.; Radin, E.L.; Nazimiec, M.; Weisser, P.A.; Curran, N.; Lewinnek, G. Role of hyaluronic acid in joint lubrication. Ann. Rheum. Dis. 1974, 33, 318-326. [CrossRef]

5. Schmidt, T.A.; Gastelum, N.S.; Nguyen, Q.T.; Schumacher, B.L.; Sah, R.L. Boundary lubrication of articular cartilage: Role of synovial fluid constituents. Arthritis Rheum 2007, 56, 882-891. [CrossRef] [PubMed] 
6. Bongaerts, J.H.H.; Rossetti, D.; Stokes, J.R. The Lubricating Properties of Human Whole Saliva. Tribol. Lett. 2007, 27, 277-287. [CrossRef]

7. Hoffman, A.S. Hydrogels for biomedical applications. Adv. Drug Deliver Rev. 2012, 64, 18-23. [CrossRef]

8. Ratner, B.D.; Hoffman, A.S. Synthetic Hydrogels for Biomedical Applications. In Hydrogels for Medical and Related Applications; American Chemical Society: Washington, DC, USA, 1976; Volume 31, pp. 1-36.

9. Peppas, N.A.; Bures, P.; Leobandung, W.; Ichikawa, H. Hydrogels in pharmaceutical formulations. Eur. J. Pharm. Biopharm. 2000, 50, 27-46. [CrossRef]

10. Wichterle, O.; Lim, D. Hydrophilic gels for biological use. Nature 1960, 185, 117. [CrossRef]

11. Lustig, S.R.; Peppas, N.A. Solute diffusion in swollen membranes. IX. Scaling laws for solute diffusion in gels. J. Appl. Polym. Sci. 1988, 36, 735-747. [CrossRef]

12. De Gennes, P.G. Dynamics of Entangled Polymer Solutions. I. The Rouse Model. Macromolecules 1976, 9, 587-593. [CrossRef]

13. De Gennes, P.-G.; Gennes, P.-G. Scaling Concepts in Polymer Physics; Cornell University Press: Ithaca, NY, USA; London, UK, 1979. [CrossRef]

14. Geissler, E.; Hecht, A.M.; Horkay, F.; Zrinyi, M. Compressional Modulus of Swollen Polyacrylamide Networks. Macromolecules 1988, 21, 2594-2599. [CrossRef]

15. Sokoloff, J.B. Theory of hydrostatic lubrication for two like-charge polymer hydrogel coated surfaces. Soft Matter 2010, 6, 3856-3862. [CrossRef]

16. Gong, J.P.; Iwasaki, Y.; Osada, Y.; Kurihara, K.; Hamai, Y. Friction of gels. 3. Friction on solid surfaces. J. Phys. Chem. B 1999, 103, 6001-6006. [CrossRef]

17. Pan, Y.S.; Xiong, D.S. Friction properties of nano-hydroxyapatite reinforced poly(vinyl alcohol) gel composites as an articular cartilage. Wear 2009, 266, 699-703. [CrossRef]

18. Chang, D.P.; Dolbow, J.E.; Zauscher, S. Switchable friction of stimulus-responsive hydrogels. Langmuir 2007, 23, 250-257. [CrossRef] [PubMed]

19. Pan, Y.S.; Xiong, D.S.; Ma, R.Y. A study on the friction properties of poly(vinyl alcohol) hydrogel as articular cartilage against titanium alloy. Wear 2007, 262, 1021-1025. [CrossRef]

20. Gong, J.P.; Kagata, G.; Osada, Y. Friction of gels. 4. Friction on charged gels. J. Phys. Chem. B 1999, 103, 6007-6014. [CrossRef]

21. Oogaki, S.; Kagata, G.; Kurokawa, T.; Kuroda, S.; Osada, Y.; Gong, J.P. Friction between like-charged hydrogels-combined mechanisms of boundary, hydrated and elastohydrodynamic lubrication. Soft Matter 2009, 5, 1879-1887. [CrossRef]

22. Gong, J.P. Friction and lubrication of hydrogels-its richness and complexity. Soft Matter 2006, 2, 544-552. [CrossRef]

23. Shoaib, T.; Espinosa-Marzal, R.M. Insight into the Viscous and Adhesive Contributions to Hydrogel Friction. Tribol. Lett. 2018, 66, 96. [CrossRef]

24. Shoaib, T.; Heintz, J.; Lopez-Berganza, J.A.; Muro-Barrios, R.; Egner, S.A.; Espinosa-Marzal, R.M. Stick-Slip Friction Reveals Hydrogel Lubrication Mechanisms. Langmuir 2018, 34, 756-765. [CrossRef] [PubMed]

25. Yashima, S.; Takase, N.; Kurokawa, T.; Gong, J.P. Friction of hydrogels with controlled surface roughness on solid flat substrates. Soft Matter 2014, 10, 3192-3199. [CrossRef] [PubMed]

26. Tominaga, T.; Kurokawa, T.; Furukawa, H.; Osada, Y.; Gong, J.P. Friction of a soft hydrogel on rough solid substrates. Soft Matter 2008, 4, 1645-1652. [CrossRef]

27. Gong, J.; Osada, Y. Gel friction: A model based on surface repulsion and adsorption. J. Chem. Phys. 1998, 109, 8062-8068. [CrossRef]

28. Simič, R.; Yetkin, M.; Zhang, K.; Spencer, N.D. Importance of Hydration and Surface Structure for Friction of Acrylamide Hydrogels. Tribol. Lett. 2020, 68, 1-12. [CrossRef]

29. Cuccia, N.L.; Pothineni, S.; Wu, B.; Mendez Harper, J.; Burton, J.C. Pore-size dependence and slow relaxation of hydrogel friction on smooth surfaces. PNAS 2020, 117, 11247-11256. [CrossRef]

30. Kim, J.; Dunn, A.C. Soft hydrated sliding interfaces as complex fluids. Soft Matter 2016, 12, 6536-6546. [CrossRef]

31. Pitenis, A.A.; Uruena, J.M.; Schulze, K.D.; Nixon, R.M.; Dunn, A.C.; Krick, B.A.; Sawyer, W.G.; Angelini, T.E. Polymer fluctuation lubrication in hydrogel gemini interfaces. Soft Matter 2014, 10, 8955-8962. [CrossRef] 
32. Shoaib, T.; Espinosa-Marzal, R.M. Influence of Loading Conditions and Temperature on Static Friction and Contact Aging of Hydrogels with Modulated Microstructures. ACS Appl. Mater. Interfaces 2019, 11, 42722-42733. [CrossRef]

33. Klein, J. Hydration lubrication. Friction 2013, 1, 1-23. [CrossRef]

34. Naka, M.H.; Morita, Y.; Ikeuchi, K. Influence of proteoglycan contents and of tissue hydration on the frictional characteristics of articular cartilage. Proc. Inst. Mech. Eng. Part H 2005, 219, 175-182. [CrossRef] [PubMed]

35. Ishikawa, Y.; Hiratsuka, K.; Sasada, T. Role of water in the lubrication of hydrogel. Wear 2006, 261, 500-504. [CrossRef]

36. Rosenberg, K.J.; Goren, T.; Crockett, R.; Spencer, N.D. Load-induced transitions in the lubricity of adsorbed poly(L-lysine)-g-dextran as a function of polysaccharide chain density. ACS Appl. Mater. Interfaces 2011, 3, 3020-3025. [CrossRef] [PubMed]

37. Klein, J.; Luckham, P. Forces between 2 Adsorbed Polyethylene Oxide Layers Immersed in a Good Aqueous Solvent. Nature 1982, 300, 429-431. [CrossRef]

38. Klein, J.; Kamiyama, Y.; Yoshizawa, H.; Israelachvili, J.N.; Fredrickson, G.H.; Pincus, P.; Fetters, L.J. Lubrication Forces between Surfaces Bearing Polymer Brushes. Macromolecules 1993, 26, 5552-5560. [CrossRef]

39. Espinosa-Marzal, R.M.; Nalam, P.C.; Bolisetty, S.; Spencer, N.D. Impact of solvation on equilibrium conformation of polymer brushes in solvent mixtures. Soft Matter 2013, 9, 4045-4057. [CrossRef]

40. Grest, G.S.; Murat, M. Structure of Grafted Polymeric Brushes in Solvents of Varying Quality-A Molecular-Dynamics Study. Macromolecules 1993, 26, 3108-3117. [CrossRef]

41. Irfachsyad, D.; Tildesley, D.; Malfreyt, P. Dissipative particle dynamics simulation of grafted polymer brushes under shear. Phys. Chem. Chem. Phys. 2002, 4, 3008-3015. [CrossRef]

42. Heuberger, M.; Drobek, T.; Spencer, N.D. Interaction forces and morphology of a protein-resistant poly(ethylene glycol) layer. Biophys. J. 2005, 88, 495-504. [CrossRef]

43. Drobek, T.; Spencer, N.D. Nanotribology of surface-grafted PEG layers in an aqueous environment. Langmuir 2008, 24, 1484-1488. [CrossRef] [PubMed]

44. Gombert, Y.; Simic, R.; Roncoroni, F.; Dbner, M.; Geue, T.; Spencer, N.D. Structuring Hydrogel Surfaces for Tribology. Adv. Mater. Interfaces 2019, 6, 1901320. [CrossRef]

45. Singh, M.K.; Ilg, P.; Espinosa-Marzal, R.M.; Kroger, M.; Spencer, N.D. Polymer Brushes under Shear: Molecular Dynamics Simulations Compared to Experiments. Langmuir 2015, 31, 4798-4805. [CrossRef]

46. McGhee, E.O.; Pitenis, A.A.; Urueña, J.M.; Schulze, K.D.; McGhee, A.J.; O’Bryan, C.S.; Bhattacharjee, T.; Angelini, T.E.; Sawyer, W.G. In Situ Measurements of Contact Dynamics in Speed-dependent Hydrogel Friction. Biotribology 2018, 13, 23-29. [CrossRef]

47. Hirayama, S.; Kurokawa, T.; Gong, J.P. Non-linear rheological study of hydrogel sliding friction in water and concentrated hyaluronan solution. Tribol. Int. 2020, 147, 106270. [CrossRef]

48. Ma, L.; Gaisinskaya-Kipnis, A.; Kampf, N.; Klein, J. Origins of hydration lubrication. Nat. Commun. 2015, 6, 6060. [CrossRef]

49. Raviv, U.; Laurat, P.; Klein, J. Fluidity of water confined to subnanometre films. Nature 2001, 413, 51-54. [CrossRef]

50. Kurokawa, T.; Tominaga, T.; Katsuyama, Y.; Kuwabara, R.; Furukawa, H.; Osada, Y.; Gong, J.P. Elastic-hydrodynamic transition of gel friction. Langmuir 2005, 21, 8643-8648. [CrossRef] [PubMed]

51. Kagata, G.; Gong, J.P.; Osada, Y. Friction of gels. 6. Effects of sliding velocity and viscoelastic responses of the network. J. Phy. Chem. B 2002, 106, 4596-4601. [CrossRef]

52. Singh, A.K.; Juvekar, V.A. Steady dynamic friction at elastomer-hard solid interface: A model based on population balance of bonds. Soft Matter 2011, 7, 10601-10611. [CrossRef]

53. Gupta, V.; Singh, A.K. Scaling laws of gelatin hydrogels for steady dynamic friction. Int. J. Mod. Phys. B 2016, 30, 1650198. [CrossRef]

54. Baumberger, T.; Caroli, C.; Ronsin, O. Self-healing slip pulses along a gel/glass interface. Phys. Rev. Lett. 2002, 88, 075509. [CrossRef] [PubMed]

55. Baumberger, T.; Caroli, C.; Ronsin, O. Self-healing slip pulses and the friction of gelatin gels. Eur. Phys. J. E Soft Matter 2003, 11, 85-93. [CrossRef]

56. Heaton, T.H. Evidence for and Implications of Self-Healing Pulses of Slip in Earthquake Rupture. Phys. Earth Planet. Inter. 1990, 64, 1-20. [CrossRef] 
57. Urueña, J.M.; Pitenis, A.A.; Nixon, R.M.; Schulze, K.D.; Angelini, T.E.; Sawyer, W.G. Mesh size control of polymer fluctuation lubrication in gemini hydrogels. Biotribology 2015, 1, 24-29. [CrossRef]

58. Reale, E.R.; Dunn, A.C. Poroelasticity-driven lubrication in hydrogel interfaces. Soft Matter 2017, 13, 428-435. [CrossRef] [PubMed]

59. Gong, J.P.; Kii, A.; Xu, J.; Hattori, Y.; Osada, Y. A possible mechanism for the substrate effect on hydrogel formation. J. Phys. Chem. B 2001, 105, 4572-4576. [CrossRef]

60. de Gennes, P.G. Polymers at an interface; a simplified view. Adv. Colloid Interface Sci. 1987, 27, $189-209$. [CrossRef]

61. Halperin, A.; Tirrell, M.; Lodge, T.P. Tethered chains in polymer microstructures. In Macromolecules: Synthesis, Order and Advanced Properties; Springer: Berlin/Heidelberg, Germany, 1992; pp. 31-71. [CrossRef]

62. Schulze, K.D.; Hart, S.M.; Marshall, S.L.; O’Bryan, C.S.; Urueña, J.M.; Pitenis, A.A.; Sawyer, W.G.; Angelini, T.E. Polymer osmotic pressure in hydrogel contact mechanics. Biotribology 2017, 11, 3-7. [CrossRef]

63. Moore, A.C.; Burris, D.L. An analytical model to predict interstitial lubrication of cartilage in migrating contact areas. J. Biomech. 2014, 47, 148-153. [CrossRef]

64. Delavoipiere, J.; Tran, Y.; Verneuil, E.; Heurtefeu, B.; Hui, C.Y.; Chateauminois, A. Friction of Poroelastic Contacts with Thin Hydrogel Films. Langmuir 2018, 34, 9617-9626. [CrossRef] [PubMed]

65. Yeung, T.; Georges, P.C.; Flanagan, L.A.; Marg, B.; Ortiz, M.; Funaki, M.; Zahir, N.; Ming, W.; Weaver, V.; Janmey, P.A. Effects of substrate stiffness on cell morphology, cytoskeletal structure, and adhesion. Cell Motil Cytoskelet. 2005, 60, 24-34. [CrossRef] [PubMed]

66. Moore, D.F. Principles and Applications of Tribology: Pergamon International Library of Science, Technology, Engineering and Social Studies: International Series in Materials Science and Technology; Elsevier: Amsterdam, The Netherlands, 2013; Volume 14.

67. Urueña, J.M.; McGhee, E.O.; Angelini, T.E.; Dowson, D.; Sawyer, W.G.; Pitenis, A.A. Normal Load Scaling of Friction in Gemini Hydrogels. Biotribology 2018, 13, 30-35. [CrossRef]

68. Ciapa, L.; Delavoipière, J.; Tran, Y.; Verneuil, E.; Chateauminois, A. Transient sliding of thin hydrogel films: The role of poroelasticity. Soft Matter 2020, 16, 6539-6548. [CrossRef] [PubMed]

69. Hamrock, B.J.; Dowson, D. Elastohydrodynamic Lubrication of Elliptical Contacts for Materials of Low Elastic-Modulus I-Fully Flooded Conjunction. J. Lubrication Tech. 1978, 100, 236-245. [CrossRef]

70. Pitenis, A.A.; Uruena, J.M.; Nixon, R.M.; Bhattacharjee, T.; Krick, B.A.; Dunn, A.C.; Angelini, T.E.; Sawyer, W.G. Lubricity from Entangled Polymer Networks on Hydrogels. J. Tribol. 2016, 138, 042102. [CrossRef]

71. Kim, J.; Dunn, A.C. Thixotropic Mechanics in Soft Hydrated Sliding Interfaces. Tribol. Lett. 2018, 66, 102. [CrossRef]

72. Rubinstein, M.; Colby, R.H. Polymer Physics; Oxford University Press: New York, NY, USA, 2003; Volume 23.

73. Schallamach, A. How does rubber slide? Wear 1971, 17, 301-312. [CrossRef]

74. Drummond, C.; Israelachvili, J.; Richetti, P. Friction between two weakly adhering boundary lubricated surfaces in water. Phys. Rev. E 2003, 67, 066110. [CrossRef]

75. Suriano, R.; Griffini, G.; Chiari, M.; Levi, M.; Turri, S. Rheological and mechanical behavior of polyacrylamide hydrogels chemically crosslinked with allyl agarose for two-dimensional gel electrophoresis. J. Mech. Behav. Biomed. Mater. 2014, 30, 339-346. [CrossRef]

76. Schulze, K.D.; Bennett, A.I.; Marshall, S.; Rowe, K.G.; Dunn, A.C. Real Area of Contact in a Soft Transparent Interface by Particle Exclusion Microscopy. J. Tribol. 2016, 138. [CrossRef]

77. Luengo, G.; Schmitt, F.-J.; Hill, R.; Israelachvili, J. Thin film rheology and tribology of confined polymer melts: Contrasts with bulk properties. Macromolecules 1997, 30, 2482-2494. [CrossRef]

78. Lu, X.; Mi, Y. Characterization of the interfacial interaction between polyacrylamide and silicon substrate by Fourier transform infrared spectroscopy. Macromolecules 2005, 38, 839-843. [CrossRef]

79. Persson, B.N.J.; Volokitin, A.I. Rubber friction on smooth surfaces. Eur. Phys. J. E 2006, 21, 69-80. [CrossRef]

80. Lee, D.W.; Banquy, X.; Israelachvili, J.N. Stick-slip friction and wear of articular joints. Proc. Natl. Acad. Sci. USA 2013, 110, E567-E574. [CrossRef] [PubMed]

81. Shoaib, T.; Yuh, C.; Wimmer, M.A.; Schmid, T.M.; Espinosa-Marzal, R.M. Nanoscale insight into the degradation mechanisms of the cartilage articulating surface preceding OA. Biomater. Sci. 2020, 8, 3944-3955. [CrossRef] [PubMed]

82. Granick, S.; Hu, H.W.; Carson, G.A. Nanorheology of Confined Polymer Melts. 2. Nonlinear Shear Response at Strongly Adsorbing Surfaces. Langmuir 1994, 10, 3867-3873. [CrossRef] 
83. Hu, H.W.; Granick, S. Viscoelastic dynamics of confined polymer melts. Science 1992, 258, 1339-1342. [CrossRef] [PubMed]

84. Drummond, C. Electric-field-induced friction reduction and control. Phys. Rev. Lett. 2012, 109, 154302. [CrossRef]

85. Rudge, R.E.D.; Scholten, E.; Dijksman, J.A. Natural and induced surface roughness determine frictional regimes in hydrogel pairs. Tribol. Int. 2020, 141, 105903. [CrossRef]

86. Pan, Y.-S.; Xiong, D.-S. Recent development on biotribology of poly (vinyl alcohol) hydrogel. Tribol. Beijing 2006, 26, 188.

87. Katta, J.K.; Marcolongo, M.; Lowman, A.; Mansmann, K.A. Friction and wear behavior of poly (vinyl alcohol)/poly (vinyl pyrrolidone) hydrogels for articular cartilage replacement. J. Biomed. Mater. Res. A 2007, 83, 471-479. [CrossRef]

88. Freeman, M.E.; Furey, M.J.; Love, B.J.; Hampton, J.M. Friction, wear, and lubrication of hydrogels as synthetic articular cartilage. Wear 2000, 241, 129-135. [CrossRef]

89. Bonyadi, S.Z.; Atten, M.; Dunn, A.C. Self-regenerating compliance and lubrication of polyacrylamide hydrogels. Soft Matter 2019, 15, 8728-8740. [CrossRef]

90. Kagata, G.; Gong, J.P.; Osada, Y. Friction of gels. 7. Observation of static friction between like-charged gels. J. Phys. Chem. B 2003, 107, 10221-10225. [CrossRef]

91. Suzuki, A.; Ishii, R.; Yamakami, Y.; Nakano, K. Surface friction of thermoresponsive poly(N-isopropylacrylamide) gels in water. Colloid Polym. Sci. 2011, 289, 561-568. [CrossRef]

92. Juvekar, V.A.; Singh, A.K. Rate and aging time dependent static friction of a soft and hard solid interface. arXiv 2016, arXiv:1602.00973.

93. Pult, H.; Tosatti, S.G.; Spencer, N.D.; Asfour, J.M.; Ebenhoch, M.; Murphy, P.J. Spontaneous Blinking from a Tribological Viewpoint. Ocul. Surf. 2015, 13, 236-249. [CrossRef] [PubMed]

94. Masen, M.A.; Veijgen, N.; Klaassen, M. Experimental Tribology of Human Skin. In Skin Biophysics; Limbert, G., Ed.; Springer International Publishing: Berlin/Heidelberg, Germany, 2019; pp. 281-295. [CrossRef]

95. Forster, H.; Fisher, J. The influence of loading time and lubricant on the friction of articular cartilage. Proc. Inst. Mech. Eng. Part H 1996, 210, 109-119. [CrossRef]

Publisher's Note: MDPI stays neutral with regard to jurisdictional claims in published maps and institutional affiliations.

(C) 2020 by the authors. Licensee MDPI, Basel, Switzerland. This article is an open access article distributed under the terms and conditions of the Creative Commons Attribution (CC BY) license (http://creativecommons.org/licenses/by/4.0/). 